\title{
Particle Interactions in Diffusiophoresis and Electrophoresis of Colloidal Spheres with Thin but Polarized Double Layers
}

\author{
Hung J. Tu and Huan J. Keh ${ }^{1}$ \\ Department of Chemical Engineering, National Taiwan University, Taipei 106-17, Taiwan, R.O.C.
}

Received March 10, 2000; accepted August 4, 2000

\begin{abstract}
Thediffusiophoretic and electrophoretic motions of two colloidal spheres in the solution of a symmetrically charged electrolyte are analyzed using a method of reflections. The particles are oriented arbitrarily with respect to the electrolyte gradient or the electric field, and they are allowed to differ in radius and in zeta potential. The thickness of the electric double layers surrounding the particles is assumed to be small relative to the radius of each particle and to the gap width between the particles, but the effect of polarization of the mobile ions in the diffuse layer is taken into account. A slip velocity of fluid and normal fluxes of solute ions at the outer edge of the thin double layer are used as the boundary conditions for the fluid phase outside the double layers. The method of reflections is based on an analysis of the electrochemical potential and fluid velocity disturbances produced by a single dielectric sphere placed in an arbitrarily varying electrolyte gradient or electric field. The solution for two-sphere interactions is obtained in expansion form correct to $O\left(r_{12}^{-7}\right)$, where $r_{12}$ is the distance between the particle centers. Our analytical results are found to be in good agreement with the available numerical solutions obtained using a boundary collocation method. On the basis of a model of statistical mechanics, the results of two-sphere interactions are used to analytically determinethe first-order effect of the volumefraction of particles of each type on the mean diffusiophoretic and eletrophoretic velocities in a bounded suspension. For a suspension of identical spheres, the mean diffusiophoretic velocity can be decreased or increased as the volume fraction of the particles is increased, while the mean electrophoretic velocity is reduced with the increase in the particle concentration. $G$ enerally speaking, the particle interaction effects can be quite significant in typical situations. $\mathcal{O} 2000$ Academic Press
\end{abstract}

Key Words: diffusiophoresis; electrophoresis; two-sphere interaction; particle concentration effect; thin but polarized double layer.

\section{INTRODUCTION}

The transport behavior of small particles in a continuous medium at low Reynolds numbers is of much fundamental and practical interest. In general, driving forces for motions of colloidal particles include concentration gradients of the particles themselves (diffusion), bulk velocities of the disperse medium

\footnotetext{
${ }^{1}$ To whom correspondence should be addressed. Fax: +886-2-2362-3040. E-mail: huan@ccms.ntu.edu.tw.
}

(convection), and gravitational or centrifugal fields (sedimentation). Problems of the colloidal transport induced by these well-known driving forces were treated extensively in the past. Another category of driving forces for the locomotions of colloidal particles involves a nonuniform imposed field (such as electric potential, temperature, or solute concentration) that interacts with the surface of each particle. The particle motions associated with this mechanism, known as "phoretic motions," have received a considerable amount of attention recently (1-3).

Perhaps the most familiar example of various phoretic motions is electrophoresis, which results from the interaction between an external electric field and the electric double layer surrounding a charged particle, and is widely used for the particle characterization and separation in a variety of colloidal and biological systems. The electrophoretic velocity $\mathbf{U}^{(0)}$ of a single nonconducting particle suspended in an unbounded electrolyte solution is simply related to the uniformly applied electric field $\mathbf{E}^{\infty}$ by the Smoluchowski equation $(4,5)$,

$$
\mathbf{U}^{(0)}=\frac{\varepsilon \zeta}{4 \pi \eta} \mathbf{E}^{\infty} .
$$

Here, $\varepsilon / 4 \pi$ is the fluid permittivity, $\eta$ is the fluid viscosity, and $\zeta$ is the zeta potential of the particle surface.

Another example of phoretic motions is diffusiophoresis, which is the migration of a particle in response to the macroscopic concentration gradient of a solute, and was applied to certain latex-particle coating processes (6). The particle moves toward or away from a region of higher solute concentration, depending on some long-range interactions between the solute molecules and the particle. In an unbounded solution of a symmetrically charged binary electrolyte with a constant concentration gradient $\nabla n^{\infty}$, the diffusiophoretic velocity of a charged particle is (7)

$$
\mathbf{U}^{(0)}=\frac{\varepsilon \zeta}{4 \pi \eta} \frac{k T}{Z e} \frac{\nabla n^{\infty}}{n^{\infty}(\mathbf{0})}\left[\frac{D_{2}-D_{1}}{D_{2}+D_{1}}+\frac{4 k T}{Z e \zeta} \ln \cosh \left(\frac{Z e \zeta}{4 k T}\right)\right] .
$$

Here, $n^{\infty}(\mathbf{0})$ is the macroscopic electrolyte concentration measured at the particle center $\mathbf{0}$ in the absence of the particle, $D_{1}$ and $D_{2}$ are the diffusion coefficients of the anion and cation, 
respectively, $Z$ is the absolute value of the valences of ions, $e$ is the charge of a proton, $k$ is the Boltzmann constant, and $T$ is the absolute temperature. For the special case of $D_{2}=D_{1}$, Eq. [1.2] predicts that the particle movement (due to chemiphoresis only) is in the direction of increasing electrolyte concentration regardless of the sign of $\zeta$ and the particle velocity is a monotonic increasing function of the magnitude of $\zeta$.

Equations [1.1] and [1.2] indicate that the electrophoretic and diffusiophoretic velocities of a dielectric particle having a uniform zeta potential on its surface are independent of the particle size and shape (and there is no rotational motion of the particle). However, their validity is based on the assumptions that the local radii of curvature of the particle are much larger than the thickness of the electric double layer at the particle surface and that the effect of polarization (relaxation effect) of the diffuse ions in the double layer due to nonuniform "osmotic" flow is negligible. In fact, important advances were made in the past in the evaluation of the phoretic velocities of colloidal particles relaxing these assumptions.

Taking the double-layer distortion from equilibrium as a perturbation, O'Brien and White (8) obtained a numerical calculation for the electrophoretic velocity of a dielectric sphere of radius $a$ in a $\mathrm{KCl}$ solution which was applicable to arbitrary values of $\zeta$ and $\kappa a$, where $\kappa^{-1}$ is the Debye screening length [equal to $\left.\left(\varepsilon k T / 8 \pi Z^{2} e^{2} n^{\infty}\right)^{\frac{1}{2}}\right]$. On the other hand, Dukhin and Derjaguin (6) obtained an analytical expression for the electrophoretic mobility of a spherical particle surrounded by a thin but polarized double layer in the solution of a symmetrically charged electrolyte. Later, O'Brien (9) generalized this analysis to the case of electrophoretic motion of a colloidal sphere in the solution containing an arbitrary combination of electrolytes. The essence of this thin-layer polarization approach is that a thin diffuse layer can still transport a significant amount of solute molecules so as to affect the solute transport outside the diffuse layer. The result for the electrophoretic velocity of a sphere with a thin but polarized double layer in a symmetric electrolyte solution can be expressed as (10)

$$
\begin{aligned}
\mathbf{U}^{(0)}= & \frac{\varepsilon \zeta}{4 \pi \eta} \mathbf{E}^{\infty}\left[\frac{1}{3}\left(2+c_{1}+c_{2}\right)\right. \\
& \left.+\frac{4 k T}{3 Z e \zeta}\left(c_{1}-c_{2}\right) \ln \cosh \left(\frac{Z e \zeta}{4 k T}\right)\right],
\end{aligned}
$$

where coefficients $c_{1}$ and $c_{2}$ are functions of $\kappa a$ defined by Eqs. [2.15a] and [2.15b]. A comparison of Eq. [1.3] with the numerical results for the $\mathrm{KCl}$ solution shows that the thin-layer polarization model is quite good over a wide range of zeta potentials when $\kappa a>20$. If $Z e|\zeta| / k T$ is small and $\kappa a$ is large, the interaction between the diffuse counterions and the particle surface is weak and the polarization of the double layer is also weak. In the limit of

$$
(\kappa a)^{-1} \exp \left(\frac{Z e|\zeta|}{2 k T}\right) \rightarrow 0
$$

$c_{1}=c_{2}=\frac{1}{2}$ and Eq. [1.3] reduces to the Smoluchowski equation [1.1]. In general, the electrophoretic velocity given by Eq. [1.3] is not a monotonic function of $\zeta$ for a finite value of $\kappa a$, unlike the prediction of Eq. [1.1].

In contrast, Prieve and Roman (11) obtained a numerical solution of the diffusiophoretic velocity over a broad range of $\zeta$ and $\kappa a$ for a charged sphere in concentration gradients of symmetric electrolytes $(\mathrm{KCl}$ or $\mathrm{NaCl}$ ) using the method of O'Brien and White (8). Recently, analytical expressions for the velocity of a dielectric sphere with a thin but polarized double layer undergoing diffusiophoresis in electrolyte solutions have also been derived $(3,12)$. The result for this diffusiophoretic velocity in a symmetric electrolyte solution is

$$
\begin{aligned}
\mathbf{U}^{(0)}= & \frac{\varepsilon \zeta}{4 \pi \eta} \frac{k T}{Z e} \frac{\nabla n^{\infty}}{n^{\infty}(\mathbf{0})}\left[\frac{2}{3}\left(\frac{D_{2}-D_{1}}{D_{2}+D_{1}}+b_{1}-b_{2}\right)\right. \\
& \left.+\frac{8 k T}{3 Z e \zeta}\left(1+b_{1}+b_{2}\right) \ln \cosh \left(\frac{Z e \zeta}{4 k T}\right)\right],
\end{aligned}
$$

where $b_{1}$ and $b_{2}$ are coefficients related to $\left(D_{2}-D_{1}\right) /\left(D_{2}+D_{1}\right)$ and $\kappa a$ defined by Eq. [2.31]. When $\kappa a>20$, the agreement between Eq. [1.5] and the numerical solution is excellent for all reasonable values of the zeta potential. In the limiting situation as given by Eq. [1.4], the effect of double-layer polarization disappears and Eq. [1.5] reduces to Eq. [1.2]. Even for the case of $D_{2}=D_{1}$, the particle velocity given by Eq. [1.5] for a finite value of $\kappa a$ may not be a monotonic function of the magnitude of $\zeta$ and its direction can reverse (toward lower electrolyte concentration) when $|\zeta|$ becomes large.

It could be found from Eqs. [1.3] and [1.5] that the effect of polarization of the diffuse layer is to decrease the particle velocity. The reason for this consequence is that the transport of the diffuse ions within the double layer reduces the local electrolyte gradient or electric field along the particle surface. Numerical calculations of Eqs. [1.3] and [1.5] show that even when $\kappa a$ is as large as 300 the effect of ion transport inside the diffuse layer cannot be ignored if $|\zeta|$ equals several $k T / e$.

In practical applications of diffusiophoresis and electrophoresis, collections of particles are usually encountered, and effects of particle interactions will be important. In the limiting case that Eqs. [1.1] and [1.2] are applicable, the normalized velocity field of the immense fluid that is dragged by a particle during diffusiophoresis is the same as for electrophoresis of the particle (1); thus, the particle interaction effects in electrophoresis under the situation of infinitesimally thin double layer (satisfying Eq. [1.4]), which were studied extensively in the past (2, 13-16), can be taken to interpret those in diffusiophoresis. An important result of these studies is that the diffusiophoretic or electrophoretic velocity of each particle is unaffected by the presence of the others if all of the particles in the unbounded solution have the same zeta potential.

When the polarization effect of diffuse ions in the double layers surrounding the particles is considered, the particle 
interaction behavior in diffusiophoresis can be quite different from that in electrophoresis, due to the fact that the particle size and some other factors are involved in each transport mechanism (3). Through the use of a boundary collocation method, the electrophoresis of multiple spheres with thin but polarized double layers in an arbitrary configuration was examined (10, 17). Recently, the thin-layer polarization model and the boundary collocation technique were also used to investigate the axisymmetric diffusiophoresis of a chain of dielectric spheres in electrolyte solutions (18). In these studies, numerical results of the electrophoretic and diffusiophoretic velocities of the particles were presented for various cases. It was found that particles with the same zeta potential do interact with one another, unlike the no-interaction results obtained in previous investigations neglecting the polarization effect of the double layers.

Until now, the interactions between particles undergoing diffusiophoresis in asymmetric configurations allowing the polarization of the diffuse ions in the double layers have not been examined. The main object of the present work is to analytically study the diffusiophoretic and electrophoretic motions of two charged colloidal spheres surrounded by polarized diffuse layers in a constant electrolyte gradient or electric field oriented arbitrarily with respect to the line through particle centers. The particles, which are freely suspended in the fluid solution, may have arbitrary radii and zeta potentials. It is assumed that the thickness of the double layers is small relative to the radius of each particle and to the surface-to-surface distance between the particles. A method of reflections is used to solve the quasisteady problem.

In the next section we consider the local electrochemical potential and fluid velocity fields produced by a single spherical particle placed in a prescribed electrolyte concentration field whose gradient is not necessarily constant over length scales comparable to the particle radius. It is shown that Eq. [1.5] (or Eq. [1.3]) also applies to a single sphere in a nonuniform electrolyte gradient (or electric field), provided that $\nabla n^{\infty}$ (or $\left.\mathbf{E}^{\infty}\right)$ is evaluated at the position of the particle center. These results are then used in Section 3 to alternately evaluate the effects of one sphere on the other in a constant applied electrolyte gradient. The translationl and angular velocities of the diffusiophoretic particles are determined in this reflection manner with an error of $O\left(r_{12}^{-8}\right)$, where $r_{12}$ is the center-to-center distance between the particles, and the results are given in Eq. [3.9]. In Section 4 our analytical results are compared with the numerical calculations obtained using the boundary collocation method for the case of two diffusiophoretic spheres oriented parallel to the undisturbed electrolyte gradient. Typical effects of the particle and electrolyte properties on the two-sphere interactions in asymmetric diffusiophoresis are discussed. In Section 5, the results of two-sphere interactions derived in Section 3 for an arbitrary configuration are applied to a theory of particle concentration effects on transport properties in dilute dispersions to obtain the effect of the volume fraction of particles of each type on the average diffusiophoretic velocities in a bounded suspension, and the general result is given in Eqs. [5.8] and [5.9]. Finally, in Section 6, the analytical solutions for the electrophoretic motions of a single dielectric sphere in an arbitrary electric field, of two spheres in a constant electric field, and of a suspension of spheres, which can be obtained by the same approach used for the diffusiophoresis in the previous sections with only minor changes, are presented. For the electrophoresis of two spheres with the line through their centers parallel and perpendicular to the applied electric field, the numerical calculations obtained using the collocation method are employed to compare our reflection results.

\section{A SINGLE CHARGED SPHERE IN AN ARBITRARY CONCENTRATION FIELD OF A SYMMETRIC ELECTROLYTE}

For the purpose of obtaining the interactions between two spherical dielectric particles undergoing diffusiophoresis in a solution of a symmetrically charged binary electrolyte by the method of reflections, it is necessary to understand the motion of a single sphere in an unbounded solution with an arbitrary concentration field $n_{\mathrm{A}}(\mathbf{x})$. The instantaneous center of the particle with uniform zeta potential $\zeta$ is positioned at $\mathbf{x}_{0}$, and the relative position vector is defined as $\mathbf{r}=\mathbf{x}-\mathbf{x}_{0}\left(=r \mathbf{e}_{\mathrm{r}}\right.$, where $\mathbf{e}_{\mathrm{r}}$ is the unit vector in the direction of $\mathbf{r}$ ). Although $\mathbf{x}_{0}$ changes with time, the problem can be dealt with as a quasi-steady state if both the Peclet number and the Reynolds number are vanishingly small. It is assumed that the polarized electric double layer surrounding the particle is thin in comparison with the particle radius $a$ (say, $\kappa a>20$ ) and that $a\left|\nabla n_{\mathrm{A}}\right| / n_{\mathrm{A}}\left(\mathbf{x}_{0}\right) \ll 1$ in the vicinity of the particle.

The fluid phase can be divided into two regions: an "inner" region defined as the thin double layer adjacent to the particle surface and an "outer" region defined as the remainder of the fluid, which is electrically neutral. In the outer region, the equations of conservation of each ionic species and the fluid momentum are the Laplace equation (3),

$$
\nabla^{2} \mu_{m}=0, \quad m=1,2,
$$

and the Stokes equations,

$$
\begin{array}{r}
\eta \nabla^{2} \mathbf{v}-\nabla p=\mathbf{0}, \\
\nabla \cdot \mathbf{v}=0 .
\end{array}
$$

In Eq. [2.1], $\mu_{m}(\mathbf{x})$ is the electrochemical potential energy of ionic species $m$ defined as

$$
\mu_{m}=\mu_{m}^{0}+k T \ln n_{m}+z_{m} e \Phi,
$$

where $\mu_{m}^{0}$ is a constant, $n_{m}(\mathbf{x})$ and $z_{m}$ are the concentration and valence, respectively, of type- $m$ ions, and $\Phi(\mathbf{x})$ is the electric potential. $m$ equal to 1 and 2 refer to the anion and cation, respectively, so $-z_{1}=z_{2}=Z>0$. In Eq. [2.2], $\mathbf{v}(\mathbf{x})$ is the fluid 
velocity and $p(\mathbf{x})$ is the dynamic pressure. Note that both $n_{m}$ and $\Phi$ also satisfy Laplace's equation.

The governing equations [2.1] and [2.2] in the outer region satisfy the following boundary conditions at the particle surface ( $r=a^{+}$, the outer edge of the thin double layer) obtained by solving for the electrochemical potentials and fluid velocity in the inner region and using a matching procedure to ensure a continuous solution in the whole fluid phase $(3,9)$,

$$
\begin{aligned}
r=a^{+}: \quad \mathbf{e}_{\mathrm{r}} \cdot \nabla \mu_{m} & =-\sum_{i=1}^{2} \beta_{m i} \mathbf{I}: \nabla_{\mathrm{s}} \nabla_{\mathrm{s}} \mu_{i}, \quad m=1,2, \\
\mathbf{v} & =\mathbf{v}_{\mathrm{S}} \equiv \mathbf{U}+\boldsymbol{\Omega} \times \mathbf{r}+\mathbf{v}^{(\mathrm{s})},
\end{aligned}
$$

where the relaxation coefficients

$\beta_{11}=\frac{1}{\kappa}\left[4\left(1+\frac{3 f_{1}}{Z^{2}}\right) \exp (\bar{\zeta}) \sinh \bar{\zeta}-\frac{12 f_{1}}{Z^{2}}(\bar{\zeta}+\ln \cosh \bar{\zeta})\right]$,

$\beta_{12}=-\frac{1}{\kappa}\left(\frac{12 f_{1}}{Z^{2}}\right) \ln \cosh \bar{\zeta}$,

$\beta_{21}=-\frac{1}{\kappa}\left(\frac{12 f_{2}}{Z^{2}}\right) \ln \cosh \bar{\zeta}$,

$$
\begin{aligned}
\beta_{22}= & \frac{1}{\kappa}\left[-4\left(1+\frac{3 f_{2}}{Z^{2}}\right) \exp (-\bar{\zeta}) \sinh \bar{\zeta}\right. \\
& \left.-\frac{12 f_{2}}{Z^{2}}(\bar{\zeta}-\ln \cosh \bar{\zeta})\right]
\end{aligned}
$$

and the apparent slip velocity

$$
\begin{aligned}
\mathbf{v}^{(\mathrm{s})}= & -\frac{\varepsilon}{2 \pi \eta} \frac{k T}{(Z e)^{2}}\left[(\bar{\zeta}+\ln \cosh \bar{\zeta}) \nabla_{\mathrm{s}} \mu_{1}\right. \\
& \left.+(-\bar{\zeta}+\ln \cosh \bar{\zeta}) \nabla_{\mathrm{s}} \mu_{2}\right] .
\end{aligned}
$$

In Eqs. [2.4]-[2.7], $\bar{\zeta}=Z e \zeta / 4 k T ; f_{m}=\varepsilon k^{2} T^{2} / 6 \pi \eta e^{2} D_{m} ; \mathbf{I}$ is the unit dyadic; $\nabla_{\mathrm{s}}=\left(\mathbf{I}-\mathbf{e}_{\mathrm{r}} \mathbf{e}_{\mathrm{r}}\right) \cdot \nabla$ denotes the gradient operator along the particle surface; $\mathbf{U}$ and $\boldsymbol{\Omega}$ are the instantaneous translational and angular velocities, respectively, of the particle to be determined. To obtain Eqs. [2.4]-[2.7], it was assumed that the concentration of each ionic species within the double layer is related to the electric potential by a Boltzmann distribution. The polarization effect arising in Eq. [2.4] allows for the diffusion and electric migration of the ions in the bulk solution into the double layer, where they are dispersed in the direction parallel to the particle surface. In the limiting situation as given by Eq. [1.4] (no polarization of the double layer), $\beta_{m i} / a=0$ and the electrochemical potentials in the outer region are independent of the dynamics within the double layer.

The electrochemical potentials far away from the particle approach the undisturbed values and the fluid is motionless there.
Thus, $\mu_{m}$ and $\mathbf{v}$ must obey

$$
\begin{array}{r}
r \rightarrow \infty: \quad \mu_{m} \rightarrow \mu_{m A}=\mu_{m}^{0}+k T\left[1-\frac{z_{m}}{Z}\left(\frac{D_{2}-D_{1}}{D_{2}+D_{1}}\right)\right] \\
m=1,2,
\end{array} \begin{array}{r}
\text { [n } n_{A} \\
\mathbf{v} \rightarrow \mathbf{0}
\end{array}
$$

The second term in the brackets of Eq. [2.8] represents the contribution from the macroscopic electric field induced by the difference of ion diffusion rates $(7,19)$. It is obvious that $\nabla^{2} n_{A}=0$ and $\nabla^{2} \mu_{m A}=0$.

A general solution to Eq. [2.1] that satisfies Eq. [2.8] is

$$
\mu_{m}=\mu_{m A}+\sum_{n=1}^{\infty}\left(\frac{a}{r}\right)^{n+1} \mathbf{S}_{n}[\cdot] \boldsymbol{\lambda}_{m n}, \quad m=1,2 .
$$

Here, the $n$ th-order polyadic $\mathbf{S}_{n}$ is a surface harmonic, defined by $\mathbf{S}_{n}=r^{n+1} \nabla^{n}\left(r^{-1}\right), \boldsymbol{\lambda}_{m n}$ are $n$ th-order polyadic constants, and the symbol [.] represents $n$ scalar products using the inner nesting convention. Substitution of Eq. [2.10] into Eq. [2.4] leads to

$$
\begin{aligned}
& \lambda_{m 1}=-\sum_{i=1}^{2} g_{m i} a\left(\nabla \mu_{i A}\right)_{0}, \\
& \lambda_{m 2}=\frac{1}{3} \sum_{i=1}^{2} h_{m i} a^{2}\left(\nabla \nabla \mu_{i A}\right)_{0},
\end{aligned}
$$

where

$$
\begin{array}{ll}
g_{11}=\frac{1}{2}\left(c_{1}^{\prime}+c_{1}\right), & g_{12}=\frac{1}{2}\left(c_{1}^{\prime}-c_{1}\right), \\
g_{21}=\frac{1}{2}\left(c_{2}^{\prime}-c_{2}\right), & g_{22}=\frac{1}{2}\left(c_{2}^{\prime}+c_{2}\right), \\
h_{11}=\frac{1}{2}\left(d_{1}^{\prime}+d_{1}\right), & h_{12}=\frac{1}{2}\left(d_{1}^{\prime}-d_{1}\right), \\
h_{21}=\frac{1}{2}\left(d_{2}^{\prime}-d_{2}\right), & h_{22}=\frac{1}{2}\left(d_{2}^{\prime}+d_{2}\right),
\end{array}
$$

with

$$
\begin{array}{r}
c_{1}=\frac{1}{2 a^{2} \Delta_{1}}\left(a^{2}-2 a \beta_{11}+3 a \beta_{12}+a \beta_{22}+2 \beta_{12} \beta_{21}-2 \beta_{11} \beta_{22}\right), \\
c_{2}=\frac{1}{2 a^{2} \Delta_{1}}\left(a^{2}-2 a \beta_{22}+3 a \beta_{21}+a \beta_{11}+2 \beta_{12} \beta_{21}-2 \beta_{11} \beta_{22}\right),
\end{array}
$$

$$
c_{1}^{\prime}=c_{1}-3 \frac{\beta_{12}}{a \Delta_{1}}, \quad c_{2}^{\prime}=c_{2}-3 \frac{\beta_{21}}{a \Delta_{1}},
$$




$$
\begin{aligned}
d_{1}= & \frac{1}{3 a^{2} \Delta_{2}}\left(a^{2}-3 a \beta_{11}+5 a \beta_{12}+2 a \beta_{22}\right. \\
& \left.+6 \beta_{12} \beta_{21}-6 \beta_{11} \beta_{22}\right) \\
d_{2}= & \frac{1}{3 a^{2} \Delta_{2}}\left(a^{2}-3 a \beta_{22}+5 a \beta_{21}+2 a \beta_{11}\right. \\
& \left.+6 \beta_{12} \beta_{21}-6 \beta_{11} \beta_{22}\right) \\
d_{1}^{\prime}= & d_{1}-\frac{10 \beta_{12}}{3 a \Delta_{2}}, \quad d_{2}^{\prime}=d_{2}-\frac{10 \beta_{21}}{3 a \Delta_{2}} \\
\Delta_{1}= & \frac{1}{a^{2}}\left(a^{2}+a \beta_{11}+a \beta_{22}-\beta_{12} \beta_{21}+\beta_{11} \beta_{22}\right) \\
\Delta_{2}= & \frac{1}{a^{2}}\left(a^{2}+2 a \beta_{11}+2 a \beta_{22}-4 \beta_{12} \beta_{21}+4 \beta_{11} \beta_{22}\right)
\end{aligned}
$$

and the subscript 0 to variables inside parentheses denotes evaluation at $\mathbf{x}=\mathbf{x}_{0}$. Substituting Eqs. [2.11] and [2.12] into Eq. [2.10], we obtain

$$
\begin{aligned}
\mu_{m}= & \mu_{m A}+\sum_{i=1}^{2}\left[g_{m i}\left(\frac{a}{r}\right)^{3} \mathbf{r} \cdot\left(\nabla \mu_{i A}\right)_{0}\right. \\
& \left.+h_{m i}\left(\frac{a}{r}\right)^{5} \mathbf{r r}:\left(\nabla \nabla \mu_{i A}\right)_{0}+O\left(\nabla \nabla \nabla \mu_{i A}\right)_{0}\right] .
\end{aligned}
$$

In the limit of Eq. [1.4], Eqs. [2.13]-[2.16] reduce to $c_{1}=$ $c_{2}=c_{1}^{\prime}=c_{2}^{\prime}=g_{11}=g_{22}=1 / 2, d_{1}=d_{2}=d_{1}^{\prime}=d_{2}^{\prime}=h_{11}=$ $h_{22}=1 / 3$, and $g_{12}=g_{21}=h_{12}=h_{21}=0$.

A solution to Eqs. [2.2], [2.5], and [2.9] for the velocity field can be constructed from Lamb's general solutions as outlined by Brenner (20). The fluid velocity is completely specified when the polyadic coefficients $\boldsymbol{\alpha}_{n}, \boldsymbol{\beta}_{n}$, and $\boldsymbol{\gamma}_{n}$ in the following formulas are calculated using the value of the velocity field on the surface of the particle $\left(\mathbf{v}_{S}\right)$ :

$$
\begin{aligned}
\mathbf{e}_{r} \cdot \mathbf{v}_{\mathrm{S}} & =\sum_{n=1}^{\infty} \boldsymbol{\alpha}_{n}[\cdot] \mathbf{S}_{n}, \\
-a \nabla \cdot \mathbf{v}_{\mathrm{S}} & =\sum_{n=1}^{\infty} \boldsymbol{\beta}_{n}[\cdot] \mathbf{S}_{n}, \\
a \mathbf{e}_{r} \cdot\left(\nabla \times \mathbf{v}_{\mathrm{S}}\right) & =\sum_{n=1}^{\infty} \gamma_{n}[\cdot] \mathbf{S}_{n} .
\end{aligned}
$$

The force and torque exerted by the surrounding fluid on the surface $r=a^{+}$are given by

$$
\begin{aligned}
& \mathbf{F}=2 \pi \eta a\left(3 \boldsymbol{\alpha}_{1}+\boldsymbol{\beta}_{1}\right), \\
& \mathbf{T}=4 \pi \eta a^{2} \boldsymbol{\gamma}_{1} .
\end{aligned}
$$

Since the particle is freely suspended in the fluid, the velocities $\mathbf{U}$ and $\boldsymbol{\Omega}$ are obtained by setting the above expressions for $\mathbf{F}$ and $\mathbf{T}$ equal to zero.
Substitution of Eq. [2.5] into the left-hand side of Eq. [2.20c] leads to

$$
\begin{aligned}
& \gamma_{1}=-2 a \Omega, \\
& \gamma_{n}=\mathbf{0} \quad \text { for } n>1 .
\end{aligned}
$$

By setting $\mathbf{T}=\mathbf{0}$ and using Eqs. [2.21b] and [2.22a], one finds

$$
\Omega=\mathbf{0},
$$

as the general result for a prescribed field $\mu_{m A}$ as long as $\nabla^{2} \mu_{m A}=0$.

The translational motion is described by the coefficients $\boldsymbol{\alpha}_{n}$ and $\boldsymbol{\beta}_{n}$. It can be obtained from Eqs. [2.5] and [2.20a] that

$$
\begin{aligned}
& \boldsymbol{\alpha}_{1}=-\mathbf{U}, \\
& \boldsymbol{\alpha}_{n}=\mathbf{0} \quad \text { for } n>1 .
\end{aligned}
$$

Finally, substituting Eq. [2.5] together with Eqs. [2.7] and [2.19] into Eq. [2.20b] and neglecting the terms of $O\left(\nabla \nabla \nabla \mu_{m A}\right)_{0}$ (only $\boldsymbol{\beta}_{1}$ and $\boldsymbol{\beta}_{2}$ are needed in the following calculations), one has

$$
\begin{aligned}
& \boldsymbol{\beta}_{1}=3 \sum_{m=1}^{2} G_{m}\left(\nabla \mu_{m A}\right)_{0}, \\
& \boldsymbol{\beta}_{2}=2 a \sum_{m=1}^{2} H_{m}\left(\nabla \nabla \mu_{m A}\right)_{0},
\end{aligned}
$$

where

$$
\begin{aligned}
G_{1}= & \frac{\varepsilon k T}{3 \pi \eta(Z e)^{2}}\left[\left(1+g_{11}-g_{21}\right) \bar{\zeta}\right. \\
& \left.+\left(1+g_{11}+g_{21}\right) \ln \cosh \bar{\zeta}\right], \\
G_{2}= & \frac{\varepsilon k T}{3 \pi \eta(Z e)^{2}}\left[\left(-1+g_{12}-g_{22}\right) \bar{\zeta}\right. \\
& \left.+\left(1+g_{12}+g_{22}\right) \ln \cosh \bar{\zeta}\right], \\
H_{1}= & -\frac{\varepsilon k T}{4 \pi \eta(Z e)^{2}}\left[\left(1+2 h_{11}-2 h_{21}\right) \bar{\zeta}\right. \\
& \left.+\left(1+2 h_{11}+2 h_{21}\right) \ln \cosh \bar{\zeta}\right], \\
H_{2}= & -\frac{\varepsilon k T}{4 \pi \eta(Z e)^{2}}\left[\left(-1+2 h_{12}-2 h_{22}\right) \bar{\zeta}\right. \\
& \left.+\left(1+2 h_{12}+2 h_{22}\right) \ln \cosh \bar{\zeta}\right] .
\end{aligned}
$$

The force-free characterstic of this problem is used with Eqs. [2.21a], [2.24a], and [2.25a], and the translational velocity of the particle is found to be

$$
\mathbf{U}=\sum_{m=1}^{2} G_{m}\left(\nabla \mu_{m A}\right)_{0} .
$$


Substitution of $\mu_{m A}$ given by Eq. [2.8] and $G_{m}$ given by Eq. [2.26] into the above equation yields

$$
\mathbf{U}=A k T\left(\nabla \ln n_{A}\right)_{0},
$$

where

$$
\begin{aligned}
A= & \frac{2 \varepsilon k T}{3 \pi \eta(Z e)^{2}}\left[\left(\frac{D_{2}-D_{1}}{D_{2}+D_{1}}+b_{1}-b_{2}\right) \bar{\zeta}\right. \\
& \left.+\left(1+b_{1}+b_{2}\right) \ln \cosh \bar{\zeta}\right],
\end{aligned}
$$

with

$$
\begin{aligned}
& b_{1}=\frac{1}{2}\left(\frac{D_{2}-D_{1}}{D_{2}+D_{1}}\right) c_{1}+\frac{1}{2} c_{1}^{\prime}, \\
& b_{2}=-\frac{1}{2}\left(\frac{D_{2}-D_{1}}{D_{2}+D_{1}}\right) c_{2}+\frac{1}{2} c_{2}^{\prime},
\end{aligned}
$$

and coefficients $c_{1}, c_{2}, c_{1}^{\prime}$, and $c_{2}^{\prime}$ are given by Eq. [2.15]. Equation [2.29] with Eq. [2.30] shows that the diffusiophoretic velocity of a spherical particle with a thin but polarized double layer in an arbitrary electrolyte concentration field is proportional to the prescribed electrolyte gradient evaluated at the particle center and is identical to Eq. [1.5] in the dependence on the properties of the surrounding solution and the particle itself.

It is understood that diffusiophoresis of a charged particle in an electrolyte solution is an electrokinetic phenomenon resulting from a linear combination of two effects: (i) chemiphoresis due to the nonuniform adsorption of counterions in the electric double layer over the particle surface, which is analogous to diffusiophoresis in nonionic media (1, 3), and (ii) electrophoresis due to the macroscopic electric field generated by the concentration gradient of the electrolyte and the difference in mobilities of the cation and anion of the electolyte, given by the second term in the brackets of Eq. [2.8]. The terms in brackets of Eq. [2.30] or [1.2] proportional to $\left(D_{2}-D_{1}\right) /\left(D_{2}+D_{1}\right)$ represent the contribution from electrophoresis, while the remainder terms are the chemiphoretic component. It will be shown in Section 6 that the electrophoretic velocity of a dielectric sphere surrounded by a thin but polarized double layer in an arbitrary electric field is also given by Eq. [1.3] with $\mathbf{E}^{\infty}$ evaluated at the position of the particle center.

The corresponding velocity field in the fluid phase surrounding the diffusiophoretic particle is determined by

$$
\mathbf{v}=\nabla \phi_{-2}+\nabla \phi_{-3}+\frac{1}{2 \eta} \mathbf{r} p_{-3}+O\left(\nabla \nabla \nabla \mu_{m A}\right)_{0}
$$

where $\phi_{-(n+1)}$ and $p_{-(n+1)}$ are solid spherical harmonic functions (21). After evaluating $\phi_{-2}, \phi_{-3}$, and $p_{-3}$ from $\boldsymbol{\alpha}_{1}, \boldsymbol{\beta}_{1}$, and $\boldsymbol{\beta}_{2}$ given by Eqs. [2.24a] and [2.25], we obtain

$$
\begin{aligned}
\mathbf{v}= & \sum_{m=1}^{2}\left\{\frac{1}{2} G_{m}\left(\frac{a}{r}\right)^{3}\left(3 \mathbf{e}_{r} \mathbf{e}_{r}-\mathbf{I}\right) \cdot\left(\nabla \mu_{m A}\right)_{0}+H_{m}\left[3\left(\frac{a}{r}\right)^{3} \mathbf{e}_{r} \mathbf{e}_{r}\right.\right. \\
& \left.\left.+\left(\frac{a}{r}\right)^{5}\left(2 \mathbf{I}-5 \mathbf{e}_{r} \mathbf{e}_{r}\right)\right] \mathbf{r}:\left(\nabla \nabla \mu_{m A}\right)_{0}+O\left(\nabla \nabla \nabla \mu_{m A}\right)_{0}\right\}
\end{aligned}
$$

For the motion of a freely suspended dielectric sphere under arbitrary imposed electrochemical potential gradient $\nabla \mu_{m A}$ (or electrolyte concentration gradient $\nabla n$ ) and velocity field $\mathbf{v}_{A}$ in an unbounded fluid, the translational and angular velocities of the particle can be obtained by linearly combining Eq. [2.23], Eq. [2.28], and the Faxen laws about $\mathbf{v}_{A}$,

$$
\begin{aligned}
& \mathbf{U}=\sum_{m=1}^{2} G_{m}\left(\nabla \mu_{m A}\right)_{0}+\left(\mathbf{v}_{A}\right)_{0}+\frac{1}{6} a^{2}\left(\nabla^{2} \mathbf{v}_{A}\right)_{0}, \\
& \boldsymbol{\Omega}=\frac{1}{2}\left(\nabla \times \mathbf{v}_{A}\right)_{0} .
\end{aligned}
$$

The superposition of the diffusiophoretic and hydrodynamic contributions in Eq. [2.34] is valid because both the governing equations and the boundary conditions are linear. Since $\nabla \mu_{m A}$ makes no contribution to the rotation of the spherical particle, Eq. [2.34b] for $\Omega$ contains only the effect of applied velocity field.

The unbounded fluid velocity caused by a force-free and torque-free rigid sphere in the applied velocity field $\mathbf{v}_{A}$ can be determined from (14)

$$
\begin{aligned}
\mathbf{v}= & \mathbf{v}_{A}-\frac{5}{2}\left(\frac{a}{r}\right)^{3} \mathbf{e}_{r} \mathbf{e}_{r} \mathbf{r}:\left(\nabla \mathbf{v}_{A}\right)_{0}-\frac{1}{2}\left(\frac{a}{r}\right)^{3} \mathbf{r} \times\left(\nabla \times \mathbf{v}_{A}\right)_{0} \\
& +\frac{1}{2}\left(\frac{a}{r}\right)^{5}\left(4 \mathbf{e}_{r} \mathbf{e}_{r} \mathbf{r}-\mathbf{r I}-\mathbf{I r}-\mathbf{e}_{\theta} \mathbf{r e}_{\theta}-\mathbf{e}_{\phi} \mathbf{r e}_{\phi}\right):\left(\nabla \mathbf{v}_{A}\right)_{0} \\
& +O\left(r^{-3} \nabla \nabla \mathbf{v}_{A}\right),
\end{aligned}
$$

where $\mathbf{e}_{r}, \mathbf{e}_{\theta}$, and $\mathbf{e}_{\phi}$ are the unit vectors in spherical coordinates. In the method-of-reflection analysis in the next section for the two-particle initeraction effects on diffusiophoresis, the particle disturbance to each reflected electrochemical potential field will be computed from Eq. [2.19], while the particle disturbances in fluid velocity caused by each reflected electrochemical potential and velocity fields will be computed from Eqs. [2.33] and [2.35], respectively.

\section{SOLUTION FOR INTERACTIONS BETWEEN TWO DIFFUSIOPHORETIC SPHERES}

In this section we consider the quasi-steady diffusiophoresis of two arbitrarily oriented dielectric spheres of radii $a_{1}$ and $a_{2}$ 
with uniform zeta potentials $\zeta_{1}$ and $\zeta_{2}$, respectively, in the solution of a symmetrically charged electrolyte. The particles are supposed to be sufficiently close to interact with each other, but sufficiently distant from boundary walls for the ambient fluid to be regarded as unbounded. Let $\mathbf{e}$ be the unit vector pointing from the center of particle 1 to the center of particle 2 and $r_{12}$ be the center-to-center distance between the particles. The prescribed electrolyte concentration gradient $\nabla n^{\infty}$ is taken to be constant over distances comparable to $r_{12}$ and the fluid at infinity is at rest. It is assumed that the thickness of the electric double layers is much smaller than the values of $a_{1}, a_{2}$, and $r_{12}-a_{1}-a_{2}$, so they do not overlap with each other. Nonetheless, the effect of polarization of the diffuse ions in thin double layers will be taken into account. The objective is to determine the correction to Eq. [1.5] for the diffusiophoretic velocity of each particle due to the presence of the other.

In the situation $\left(a_{1}+a_{2}\right) / r_{12} \ll 1$, a method of reflections $(14,22)$ is used to solve the two-sphere problem. Because of the linear characteristic of the governing equations [2.1] and [2.2], as well as the boundary conditions represented by Eqs. [2.4], [2.5], [2.8], and [2.9], the solution of the ionic electrochemical potentials and fluid velocity in the outer region can be decomposed into a sum of fields, which depend on increasing powers of $r_{12}^{-1}$,

$$
\begin{aligned}
\mu_{m} & =\mu_{m 1}^{(1)}+\mu_{m 2}^{(2)}+\mu_{m 1}^{(3)}+\mu_{m 2}^{(4)}+\cdots, \quad m=1,2 \\
\mathbf{v} & =\mathbf{v}_{1}^{(1)}+\mathbf{v}_{2}^{(2)}+\mathbf{v}_{1}^{(3)}+\mathbf{v}_{2}^{(4)}+\cdots,
\end{aligned}
$$

where subscripts 1 and 2 represent the reflections from particle 1 and particle 2, respectively, and the superscript ( $i$ ) denotes the $i$ th reflection from either particle surface. Hence, the particles' translational and angular velocities can also be expressed in the form of a series,

$$
\begin{aligned}
& \mathbf{U}_{1}=\mathbf{U}_{1}^{(0)}+\mathbf{U}_{1}^{(2)}+\mathbf{U}_{1}^{(4)}+\cdots, \\
& \boldsymbol{\Omega}_{1}=\boldsymbol{\Omega}_{1}^{(0)}+\boldsymbol{\Omega}_{1}^{(2)}+\boldsymbol{\Omega}_{1}^{(4)}+\cdots, \\
& \mathbf{U}_{2}=\mathbf{U}_{2}^{(1)}+\mathbf{U}_{2}^{(3)}+\mathbf{U}_{2}^{(5)}+\cdots, \\
& \boldsymbol{\Omega}_{2}=\boldsymbol{\Omega}_{2}^{(1)}+\boldsymbol{\Omega}_{2}^{(3)}+\boldsymbol{\Omega}_{2}^{(5)}+\cdots,
\end{aligned}
$$

where $\mathbf{U}_{1}^{(i)}$ and $\boldsymbol{\Omega}_{1}^{(i)}$ are related to $\mu_{m 2}^{(i)}$ and $\mathbf{v}_{2}^{(i)}$ by Eq. [2.34] for $i=2,4,6, \ldots$, while $\mathbf{U}_{2}^{(i)}$ and $\boldsymbol{\Omega}_{2}^{(i)}$ are related to $\mu_{m 1}^{(i)}$ and $\mathbf{v}_{1}^{(i)}$ for $i=1,3,5, \ldots$. According to Eqs. [2.28], [2.23], and [2.8], the unperturbed linear concentration field of the electrolyte gives

$$
\begin{aligned}
\mathbf{U}_{1}^{(0)} & =\sum_{m=1}^{2}\left(G_{m}\right)_{1} \nabla \mu_{m}^{\infty}, \\
\boldsymbol{\Omega}_{1}^{(0)} & =\mathbf{0},
\end{aligned}
$$

where

$$
\mu_{m}^{\infty}=\mu_{m}^{0}+k T\left[1-\frac{z_{m}}{Z}\left(\frac{D_{2}-D_{1}}{D_{2}+D_{1}}\right)\right] \ln n^{\infty} .
$$

Hereinafter, we use $\left(g_{m i}\right)_{j},\left(G_{m}\right)_{j},\left(H_{m}\right)_{j}, A_{j}$, and $\left(b_{m}\right)_{j}$ to represent the values of $g_{m i}, G_{m}, H_{m}, A$, and $b_{m}$, respectively, defined by Eqs. [2.13], [2.26], [2.27], [2.30], and [2.31] for the particle $j(j=1$ or 2$)$.

The initial electrochemical potential gradient $\nabla \mu_{m 1}^{(1)}$ and velocity field $\mathbf{v}_{1}^{(1)}$, which correspond to the diffusiophoresis of particle 1 isolated in an unbounded fluid under the prescribed field $\nabla \mu_{m}^{\infty}$, are easily obtained from Eqs. [2.19] and [2.33] for $r_{1} \geq a_{1}$ as

$$
\begin{aligned}
\nabla \mu_{m 1}^{(1)} & =\nabla \mu_{m}^{\infty}-\sum_{i=1}^{2}\left(g_{m i}\right)_{1}\left(\frac{a_{1}}{r_{1}}\right)^{3}\left(3 \frac{\mathbf{r}_{1} \mathbf{r}_{1}}{r_{1}^{2}}-\mathbf{I}\right) \cdot \nabla \mu_{i}^{\infty} \\
\mathbf{v}_{1}^{(1)} & =\frac{1}{2} \sum_{m=1}^{2}\left(G_{m}\right)_{1}\left(\frac{a_{1}}{r_{1}}\right)^{3}\left(3 \frac{\mathbf{r}_{1} \mathbf{r}_{1}}{r_{1}^{2}}-\mathbf{I}\right) \cdot \nabla \mu_{m}^{\infty}
\end{aligned}
$$

where $\mathbf{r}_{j}$ is the position vector relative to the center of particle $j$ and $r_{j}=\left|\mathbf{r}_{j}\right|$. Note that $\mathbf{v}_{1}^{(1)}$ is irrotational and satisfies Laplace's equation. The contributions of $\nabla \mu_{m 1}^{(1)}$ and $\mathbf{v}_{1}^{(1)}$ to the velocity of particle 2 (with the center at position $\mathbf{r}_{1}=r_{12} \mathbf{e}$ ) are determined from Eq. [2.34] taking $\mu_{m A}=\mu_{m 1}^{(1)}$ and $\mathbf{v}_{A}=\mathbf{v}_{1}^{(1)}$. Thus,

$$
\begin{aligned}
\mathbf{U}_{2}^{(1)}= & \sum_{m=1}^{2}\left\{\left(G_{m}\right)_{2} \nabla \mu_{m}^{\infty}+\left[\frac{1}{2}\left(G_{m}\right)_{1}(3 \mathbf{e e}-\mathbf{I}) \cdot \nabla \mu_{m}^{\infty}\right.\right. \\
& \left.\left.-\sum_{i=1}^{2}\left(G_{m}\right)_{2}\left(g_{m i}\right)_{1}(3 \mathbf{e e}-\mathbf{I}) \cdot \nabla \mu_{i}^{\infty}\right]\left(\frac{a_{1}}{r_{12}}\right)^{3}\right\},
\end{aligned}
$$

$\boldsymbol{\Omega}_{2}^{(1)}=\mathbf{0}$.

Equation [3.6a] indicates that the effect of particle interaction in diffusiophoresis is $O\left(r_{12}^{-3}\right)$.

The first reflected electrochemical potential gradient field from particle 2 can be derived from using Eqs. [2.19] and [3.5a], while the first reflected velocity field from particle 2 can be determined from Eqs. [2.33], [2.35], and [3.5]. The results are

$$
\begin{aligned}
\nabla \mu_{m 2}^{(2)}= & -\sum_{i=1}^{2}\left[\left(g_{m i}\right)_{2}\left(\frac{a_{2}}{r_{2}}\right)^{3}\left(3 \frac{\mathbf{r}_{2} \mathbf{r}_{2}}{r_{2}^{2}}-\mathbf{I}\right) \cdot\left(\nabla \mu_{i 1}^{(1)}\right)_{\mathbf{r}_{1}=r_{12} \mathbf{e}}\right. \\
& \left.+O\left(r_{2}^{-4} \nabla \nabla \mu_{i 1}^{(1)}+r_{2}^{-3} \nabla \nabla \nabla \mu_{i 1}^{(1)}\right)\right], \\
\mathbf{v}_{2}^{(2)}= & \sum_{m=1}^{2}\left[\frac{1}{2}\left(G_{m}\right)_{2}\left(\frac{a_{2}}{r_{2}}\right)^{3}\left(3 \frac{\mathbf{r}_{2} \mathbf{r}_{2}}{r_{2}^{2}}-\mathbf{I}\right) \cdot\left(\nabla \mu_{m 1}^{(1)}\right)_{\mathbf{r}_{1}=r_{12} \mathbf{e}}\right. \\
& +3\left(H_{m}\right)_{2}\left(\frac{a_{2}}{r_{2}}\right)^{3} \frac{\mathbf{r}_{2} \mathbf{r}_{2} \mathbf{r}_{2}}{r_{2}^{2}}:\left(\nabla \nabla \mu_{m 1}^{(1)}\right)_{\mathbf{r}_{1}=r_{12} \mathbf{e}}
\end{aligned}
$$




$$
\begin{aligned}
& \left.+O\left(r_{2}^{-4} \nabla \nabla \mu_{m 1}^{(1)}+r_{2}^{-3} \nabla \nabla \nabla \mu_{m 1}^{(1)}\right)\right] \\
& -\frac{5}{2}\left(\frac{a_{2}}{r_{2}}\right)^{3} \frac{\mathbf{r}_{2} \mathbf{r}_{2} \mathbf{r}_{2}}{r_{2}^{2}}:\left(\nabla \mathbf{v}_{1}^{(1)}\right)_{\mathbf{r}_{1}=r_{12} \mathbf{e}} \\
& +O\left(r_{2}^{-4} \nabla \mathbf{v}_{1}^{(1)}+r_{2}^{-3} \nabla \nabla \mathbf{v}_{1}^{(1)}\right) .
\end{aligned}
$$

Substituting Eq. [3.7] into Eq. [2.34] with $\mu_{m A}=\mu_{m 2}^{(2)}$ and $\mathbf{v}_{A}=$ $\mathbf{v}_{2}^{(2)}$, one obtains the contribution to the velocity of particle 1 due to the reflected fields from particle 2 ,

$$
\begin{aligned}
\mathbf{U}_{1}^{(2)}= & \sum_{m=1}^{2}\left[\frac{1}{2}\left(G_{m}\right)_{2}(3 \mathbf{e e}-\mathbf{I}) \cdot \nabla \mu_{m}^{\infty}\right. \\
& \left.-\sum_{i=1}^{2}\left(G_{m}\right)_{1}\left(g_{m i}\right)_{2}(3 \mathbf{e e}-\mathbf{I}) \cdot \nabla \mu_{i}^{\infty}\right]\left(\frac{a_{2}}{r_{12}}\right)^{3} \\
& +\sum_{m=1}^{2}\left\{\sum_{i=1}^{2}\left[-\frac{1}{2}\left(G_{m}\right)_{2}\left(g_{m i}\right)_{1}+\left(G_{m}\right)_{1}\left(g_{m i}\right)_{1}\left(g_{m i}\right)_{2}\right]\right. \\
& \times(3 \mathbf{e e}+\mathbf{I}) \cdot \nabla \mu_{i}^{\infty}-18 \sum_{i=1}^{2}\left(H_{m}\right)_{2}\left(g_{m i}\right)_{1} \mathbf{e e} \cdot \nabla \mu_{i}^{\infty} \\
& \left.-\frac{15}{2}\left(G_{m}\right)_{1} \mathbf{e e} \cdot \nabla \mu_{m}^{\infty}\right\} \frac{a_{1}^{3} a_{2}^{3}}{r_{12}^{6}}+O\left(r_{12}^{-8}\right), \\
\mathbf{\Omega}_{1}^{(2)}= & -\sum_{m=1}^{2}\left[9 \sum_{i=1}^{2}\left(H_{m}\right)_{2}\left(g_{m i}\right)_{1} \mathbf{e} \times \nabla \mu_{i}^{\infty}\right. \\
& \left.+\frac{15}{4}\left(G_{m}\right)_{1} \mathbf{e} \times \nabla \mu_{m}^{\infty}\right] \frac{a_{1}^{3} a_{2}^{3}}{r_{12}^{7}}+O\left(r_{12}^{-9}\right) .
\end{aligned}
$$

The $O\left(r_{12}^{-8}\right)$ and $O\left(r_{12}^{-9}\right)$ interactions in Eq. [3.8] could be obtained by more detailed calculations of $\nabla \mu_{m 2}^{(2)}$ and $\mathbf{v}_{2}^{(2)}$ and their derivatives at the position $\mathbf{r}_{2}=-r_{12} \mathbf{e}$ (the center of particle 1), but the numerical significance would be small unless the two particles are almost in contact.

Obviously, $\mathbf{U}_{1}^{(4)}$ and $\Omega_{1}^{(4)}$ will be of the orders $O\left(r_{12}^{-9}\right)$ and $O\left(r_{12}^{-10}\right)$, respectively. With the addition of Eqs. [3.3] and [3.8] after the substitution of Eq. [3.4], the translational and angular velocities of particle 1 can be expressed as

$$
\begin{aligned}
\mathbf{U}_{1}= & A_{1} \frac{k T}{n^{\infty}(\mathbf{0})} \nabla n^{\infty}+\frac{k T}{n^{\infty}(\mathbf{0})}\left[\frac{1}{2} A_{2}-2 \sum_{m=1}^{2}\left(G_{m}\right)_{1}\left(b_{m}\right)_{2}\right] \\
& \times\left(\frac{a_{2}}{r_{12}}\right)^{3}(3 \mathbf{e e}-\mathbf{I}) \cdot \nabla n^{\infty}+\frac{k T}{n^{\infty}(\mathbf{0})} \\
& \times\left\{\sum_{m=1}^{2}\left[-\left(G_{m}\right)_{2}\left(b_{m}\right)_{1}+2 \sum_{i=1}^{2}\left(G_{m}\right)_{1}\left(g_{m i}\right)_{2}\left(b_{i}\right)_{1}\right]\right.
\end{aligned}
$$

$$
\begin{aligned}
& \left.\times(3 \mathbf{e e}+\mathbf{I})-\left[36 \sum_{m=1}^{2}\left(H_{m}\right)_{2}\left(b_{m}\right)_{1}+\frac{15}{2} A_{1}\right] \mathbf{e e}\right\} \\
& \times \frac{a_{1}^{3} a_{2}^{3}}{r_{12}^{6}} \cdot \nabla n^{\infty}+O\left(r_{12}^{-8}\right) \\
\mathbf{\Omega}_{1}= & \frac{k T}{n^{\infty}(\mathbf{0})}\left[18 \sum_{m=1}^{2}\left(H_{m}\right)_{2}\left(b_{m}\right)_{1}+\frac{15}{4} A_{1}\right] \frac{a_{1}^{3} a_{2}^{3}}{r_{12}^{7}} \mathbf{e} \\
& \times \nabla n^{\infty}+O\left(r_{12}^{-9}\right)
\end{aligned}
$$

$\mathbf{U}_{2}$ and $\boldsymbol{\Omega}_{2}$, the velocities of particle 2, can be obtained from the above closed-form formulas by interchanging the subscripts 1 and 2 in all variables and replacing e by $-\mathbf{e}$. As expected, both particles will move with the velocity that would exist in the absence of the other (without rotation) for any orientation of the particles as $r_{12} \rightarrow \infty$. Equation [3.9a] indicates that the direction of diffusiophoresis of each sphere is deflected by the other, unless the electrolyte gradient is prescribed either parallel or perpendicular to the line through the particle centers.

When the gap between the two diffusiophoretic particles approaches zero (but is still large relative to the Debye screening length so that their double layers do not overlap), i.e., $\left(a_{1}+a_{2}\right) / r_{12} \rightarrow 1$, the expansions given by Eq. [3.9] may not be sufficiently accurate, and the lubrication theory could be a possible method of getting semianalytical approximations for the particle velocities under this situation $(23,24)$. These nearcontact interaction results would be important for the prediction of pairwise aggregation rates in a suspension of particles undergoing diffusiophoresis (25).

\section{DISCUSSION ON INTERACTIONS BETWEEN TWO DIFFUSIOPHORETIC SPHERES}

The interaction between two charged spheres in a concentration gradient of an electrolyte, given by Eq. [3.9], results from two phenomena: each particle disturbs the local concentration or electrochemical potential field experienced by the other, and the movement of each particle (with an apparent slip velocity at the surface) generates a fluid velocity field that convects and rotates the other. The leading term of the interaction for particle translation is $O\left(r_{12}^{-3}\right)$, because both electrochemical potential gradient and velocity disturbances in the fluid phase produced by a single diffusiophoretic sphere decay like $r^{-3}$, as shown in Eqs. [2.19] and [2.33]. For two diffusiophoretic spheres that allow free rotation, the leading term of the angular velocity is of $O\left(r_{12}^{-7}\right)$.

The exact (numerical) solution for the problem of diffusiophoresis of two dielectric spheres parallel to the line through their centers was obtained by using a boundary collocation method (18). Figure 1 gives a comparison of our asymptotic results of the particle velocities (normalized by their undisturbed values given by Eq. [1.5]) calculated from the method of reflections (illustrated by curves) with this exact solution 

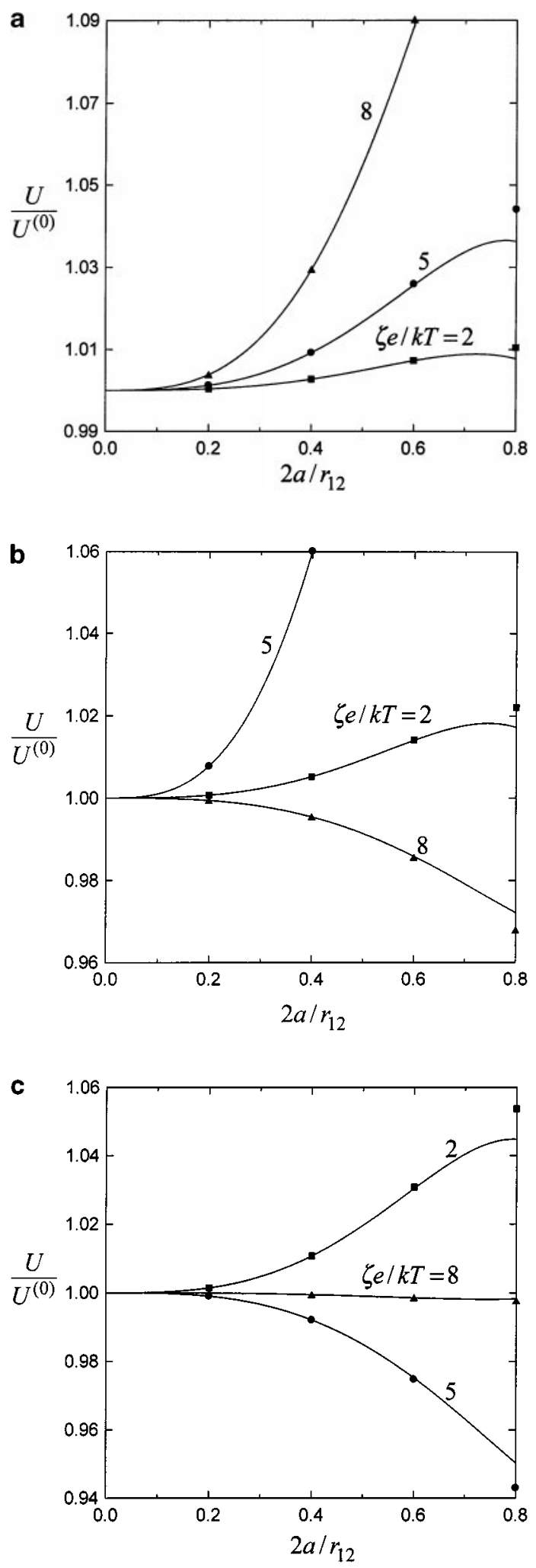

FIG . 1. Normalized diffusiophoretic velocities of two identical spheres with the line through their centers aligned with the imposed electrolyte gradient versus the separation parameter $2 a / r_{12}$ with $\zeta e / k T$ as a parameter for the case of $f_{1}=f_{2}=0.2$ and $\kappa a=100$ : (a) $Z=1$; (b) $Z=2$; (c) $Z=3$. The points represent the exact collocation solutions. (shown by points). For simplicity, only the case of two identical spheres $\left(a_{1}=a_{2}=a, \zeta_{1}=\zeta_{2}=\zeta, U_{1}^{(0)}=U_{2}^{(0)}=U^{(0)}\right.$, where $\mathbf{U}_{j}^{(0)}=U_{j}^{(0)} \mathbf{d}$ and $\mathbf{d}$ is the unit vector in the direction of $\nabla n^{\infty}$ ) with $f_{1}=f_{2}=0.2$ and $\kappa a=100$ is presented. In the case of identical spheres, the particles will migrate at the same velocity ( $U_{1}=U_{2}=U$, where $\mathbf{U}_{j}=U_{j} \mathbf{d}$ ). It is found in Fig. 1 that the predictions of $U / U^{(0)}$ from the asymptotic approximation for various values of $Z$ and $\zeta e / k T$ are in good agreement with those of the exact solution. The errors in diffusiophoretic velocities are less than $0.28 \%$ for cases $2 a / r_{12} \leq 0.6$ or $3.5 \%$ for cases $2 a / r_{12} \leq 0.8$, indicating that the higher-order terms such as $O\left(r_{12}^{-8}\right)$ in Eq. [3.9] are not important unless the particles are nearly touching. For the cases of two spheres differing in size and/or in zeta potential, or under the situation $f_{1} \neq f_{2}$, Eq. [3.9a] can also be found to agree well with the exact solution.

The plots of the numerical values of $U_{i} / U_{i}^{(0)}$ versus parameters $\zeta e / k T$ and $\kappa a_{i}$ for various cases of two charged spheres undergoing diffusiophoresis along the line through their centers were presented by Keh and Luo (18). Readers are suggested to refer to their work for details of the particle interactions in this axisymmetric configuration. The translational and rotational velocities for various cases of two identical spheres (with $\Omega_{1}=-\Omega_{2}=\Omega$, where $\Omega_{j}=\Omega_{j} \mathbf{e} \times \mathbf{d}$ ) undergoing diffusiophoresis perpendicular to the line through their centers evaluated from Eq. [3.9] are shown in Figs. 2-6. For the asymmetric configuration, there is no numerical solution available in the literature to make a comparison. In Figs. 2 and 3 (for cases of $f_{1}=f_{2}$ and $f_{1} \neq f_{2}$, respectively), the velocities of a particle normalized by the value that prevails in the absence of the other are plotted versus the separation parameter $2 a / r_{12}$ for various values of $Z$ and $\zeta e / k T$. It can be seen that the effect of particle interactions on the normalized diffusiophoretic velocities is increased with the increase in $2 a / r_{12}$. However, each sphere can be speeded up or slowed down (and rotated in either direction) by the movement of the other with the decrease of the separation distance, depending on the values of the relevant factors. In general, if the translational velocities of the two spheres undergoing diffusiophoresis normal to their line of centers are enhanced (or reduced) by the proximity of each other, then the angular velocity $\Omega$ (and diffusiophoretic velocities along their line of centers, which are shown in Fig. 1) will be decreased (or increased). For some typical cases, the effect of particle interactions can be quite significant as the particles get close to each other. Note that the situations associated with Figs. $2 \mathrm{a}$ and $3 \mathrm{a}$ are close to those of diffusiophoresis in the aqueous solutions of $\mathrm{KCl}$ and $\mathrm{NaCl}$, respectively.

The normalized diffusiophoretic velocities of two identical spheres in the direction normal to the line through their centers are plotted as a function of their dimensionless zeta potential at different values of $\kappa a(20,100$, and $\infty)$ and $Z$ in Fig. 4 for a case in which the cation and anion mobilities are equal (with $f_{1}=f_{2}=0.2$ and $2 a / r_{12}=0.6$ ). Only the results at positive zeta potentials are displayed in this figure since, for $D_{2}=D_{1}$, 

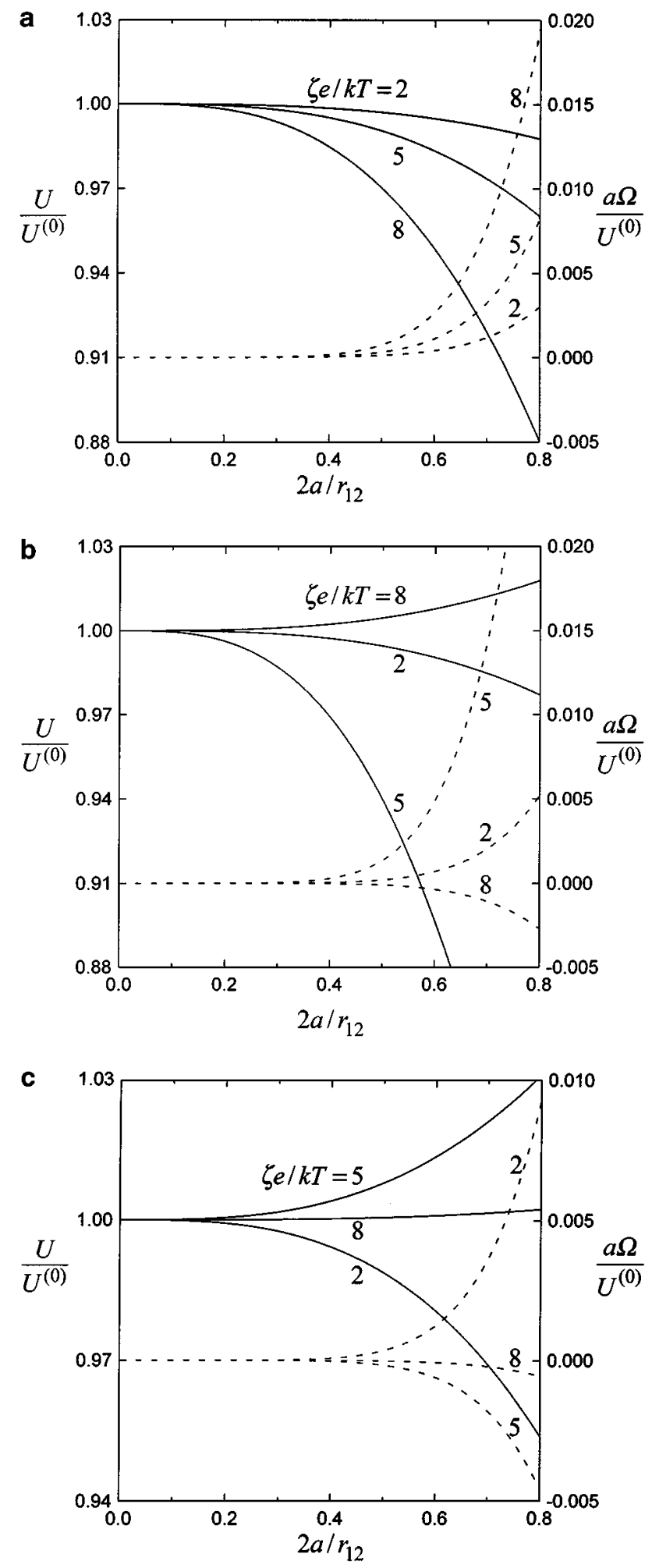

FIG. 2. Normalized translational (solid curves) and rotational (dashed curves) velocities of two identical spheres undergoing diffusiophoresis perpendicular to the line through their centers versus the separation parameter $2 a / r_{12}$ with $\zeta e / k T$ as a parameter for the case of $f_{1}=f_{2}=0.2$ and $\kappa a=100$ : (a) $Z=1$; (b) $Z=2$; (c) $Z=3$.
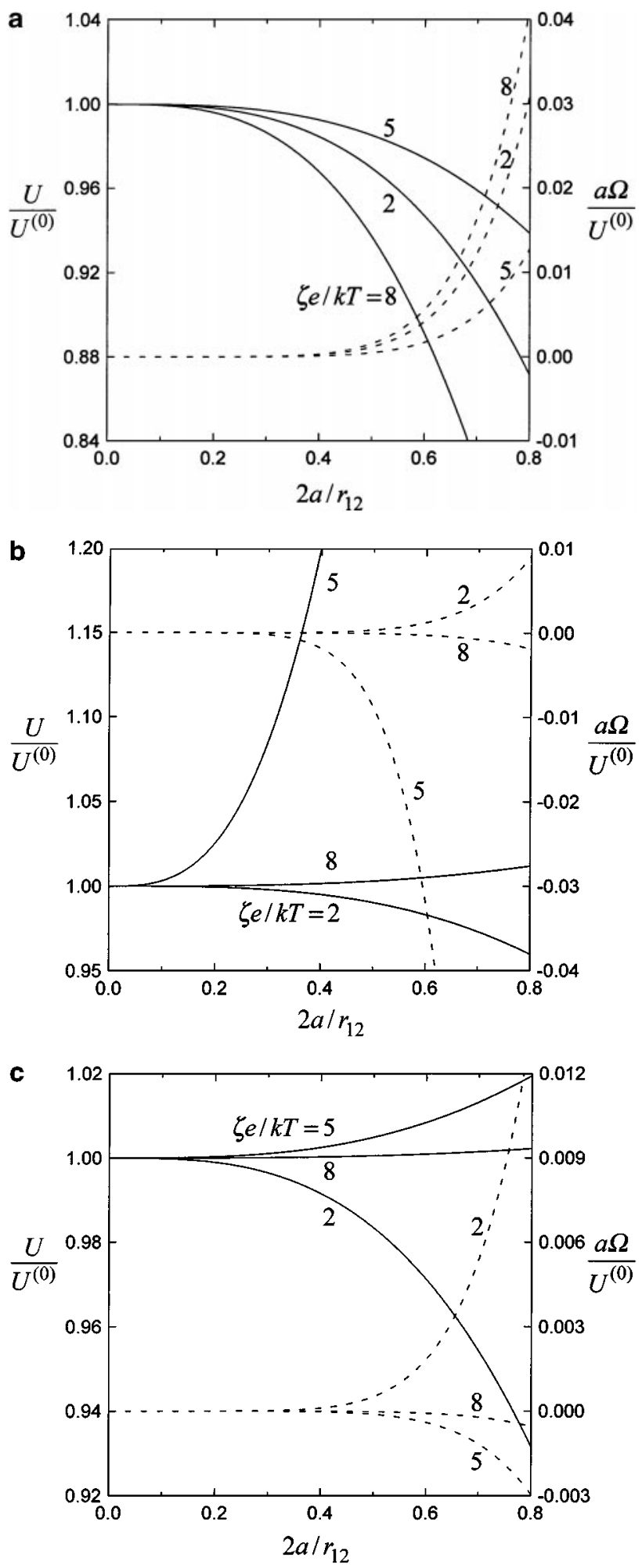

FIG. 3. Normalized translational (solid curves) and rotational (dashed curves) velocities of two identical spheres undergoing diffusiophoresis perpendicular to the line through their centers versus the separation parameter $2 a / r_{12}$ with $\zeta e / k T$ as a parameter for the case of $\left(D_{2}-D_{1}\right) /\left(D_{2}+D_{1}\right)=-0.2, f_{1}=$ 0.2 , and $\kappa a=100$ : (a) $Z=1$; (b) $Z=2$; (c) $Z=3$. 

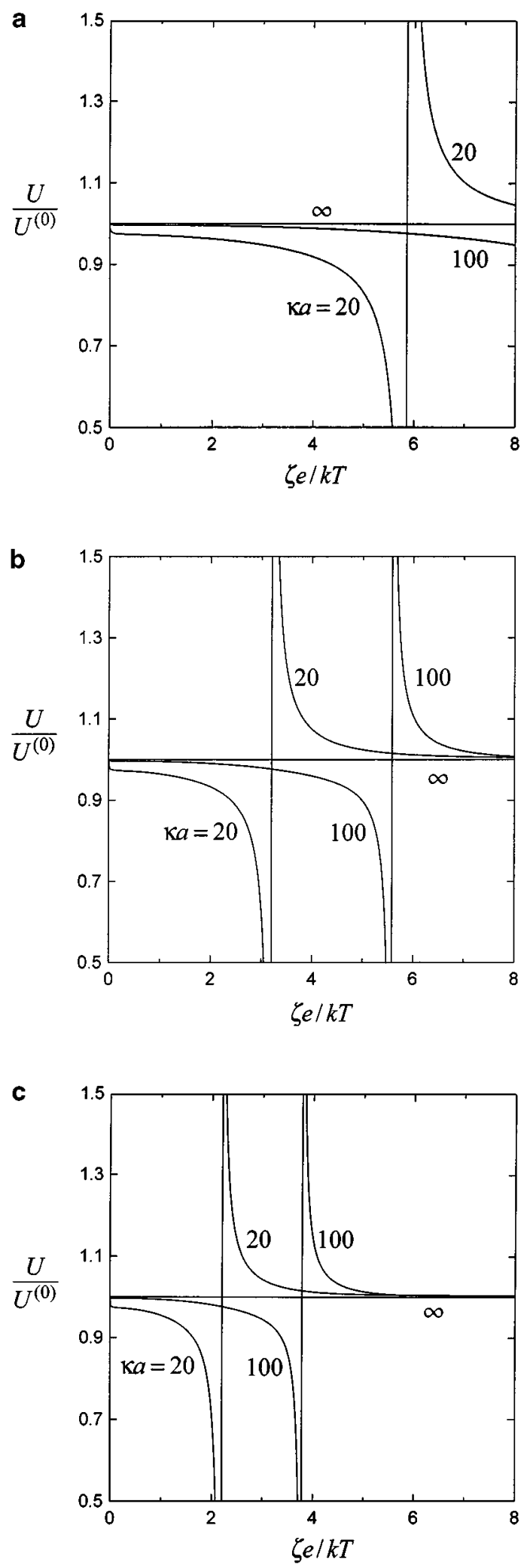

FIG . 4. Normalized translational velocities of two identical spheres undergoing diffusiophoresis perpendicular to the line through their centers versus the dimensionless zeta potential $\zeta e / k T$ with $\kappa a$ as a parameter for the case of $f_{1}=f_{2}=0.2$ and $2 a / r_{12}=0.6$ : (a) $Z=1$; (b) $Z=2$; (c) $Z=3$. the induced macroscopic electric field vanishes and the particle velocities, which are due to the chemiphoretic effect only, will be an even function of the zeta potential. When the value of $Z e \zeta / k T$ is small (say, <6), the normalized diffusiophoretic mobility of each particle is a monotonic decreasing function of $Z e \zeta / k T$ for a finite value of $\kappa a$. Also, this mobility is smaller with smaller $\kappa a$. However, when the value of $Z e \zeta / k T$ is increased, a minimum and a maximum of the normalized mobility would appear. As $Z$ increases or $\kappa a$ decreases, the extremes occur at smaller zeta potentials. Note that the abrupt variation of the normalized particle velocity near these extremes is due to the fact that the direction of the undisturbed velocity $\mathbf{U}^{(0)}$ reverses and its magnitude is small over there (refer to Refs. 3 and 11 ), while the interacted particle velocity $\mathbf{U}_{j}$ does not reverse synchronously.

The normalized diffusiophoretic velocities of two identical spheres perpendicular to their line of centers as a function of $\zeta e / k T$ at different valus of $\kappa a$ and $Z$ for a case in which the cation and anion have different diffusion coefficients $\left[\left(D_{2}-\right.\right.$ $\left.D_{1}\right) /\left(D_{2}+D_{1}\right)=-0.2$ with $f_{1}=0.2$ and $\left.2 a / r_{12}=0.6\right]$ are depicted in Fig. 5. In this case, both the chemiphoretic and the electrophoretic effects contribute to the particles' movement and the net diffusiophoretic velocities are neither an even nor an odd function of $\zeta$. It can be seen that the normalized diffusiophoretic mobility of each sphere is not a monotonic function of $\zeta e / k T$. In general, no simple rule could appropriately describe the particle interactions. Whether the diffusiophoretic mobility is increased or decreased depends on the combination of $\zeta e / k T, \kappa a, Z, f_{1}$, and $f_{2}$. Since the undisturbed particle velocity $\mathbf{U}^{(0)}$ in the given range of $\zeta e / k T$ (from -8 to 8 ) can reverse its direction more times in diffusiophoresis with $D_{2} \neq D_{1}$ than in pure chemiphoresis, there are more extremes of the normalized particle mobility in Fig. 5 than in Fig. 4.

In Fig. 6, the diffusiophoretic velocities of two identical spheres normal to their line of centers are plotted versus $\kappa a$ in the range from 20 to $10^{5}$ for a case of $D_{2}=D_{1}$. It is shown that there will be no particle interaction in diffusiophoresis as long as the value of $\kappa a$ approaches infinity (the diffusiophoretic mobilities of the particles will be equal to the value calculated by Eq. [1.2] ignoring the polarization effect of the double layer). For the case $Z=1$, the particle interaction in general is weakened steadily as $\kappa a$ becomes large gradually. However, when the value of $Z e \zeta / k T$ gets large (say, $>6$ ), there can be a minimum and a maximum of the particle interaction occurring at some value of $\kappa a$ for a given value of $\zeta e / k T$. If the particles are charged more highly (with greater magnitude in zeta potential) or the counterions have a larger absolute value of valence, the locations of these maximal particle interactions will shift toward large $\kappa a$; that means larger values of $\kappa a$ are required to make the assumption of $\kappa a \rightarrow \infty$ valid. This behavior is in accordance with the limiting requirement given by Eq. [1.4]. Although only the situation of $D_{2}=D_{1}$ is displayed in Fig. 6, the plot of $U / U^{(0)}$ versus $\kappa a$ for cases with $D_{2} \neq D_{1}$ will indicate a similar outcome. 

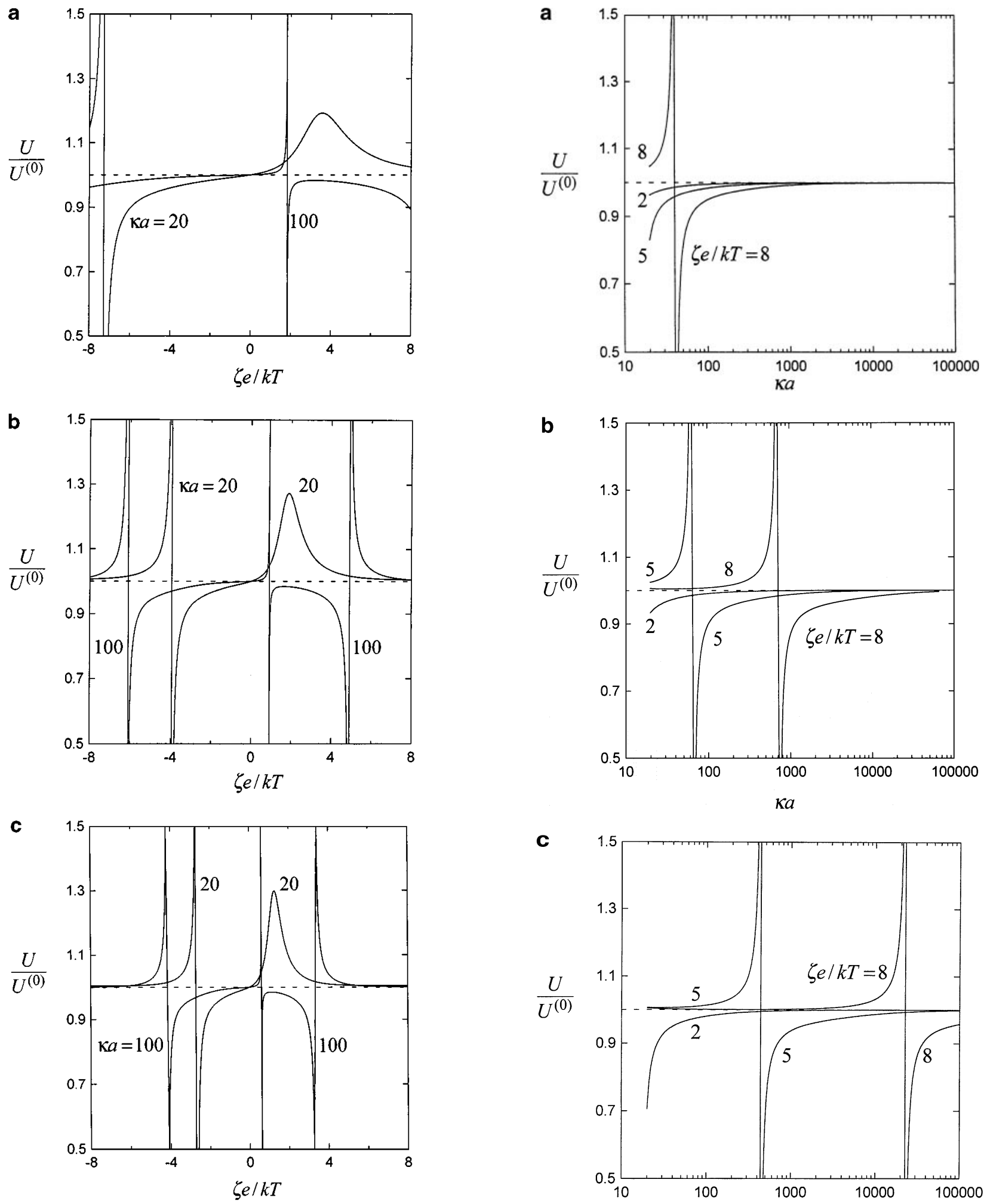

FIG. 5. Normalized translational velocities of two identical spheres undergoing diffusiophoresis perpendicular to the line through their centers versus the dimensionless zeta potential $\zeta e / k T$ with $\kappa a$ as a parameter for the case of $\left(D_{2}-D_{1}\right) /\left(D_{2}+D_{1}\right)=-0.2, f_{1}=0.2$, and $2 a / r_{12}=0.6$ : (a) $Z=1$; (b) $Z=2$; (c) $Z=3$.

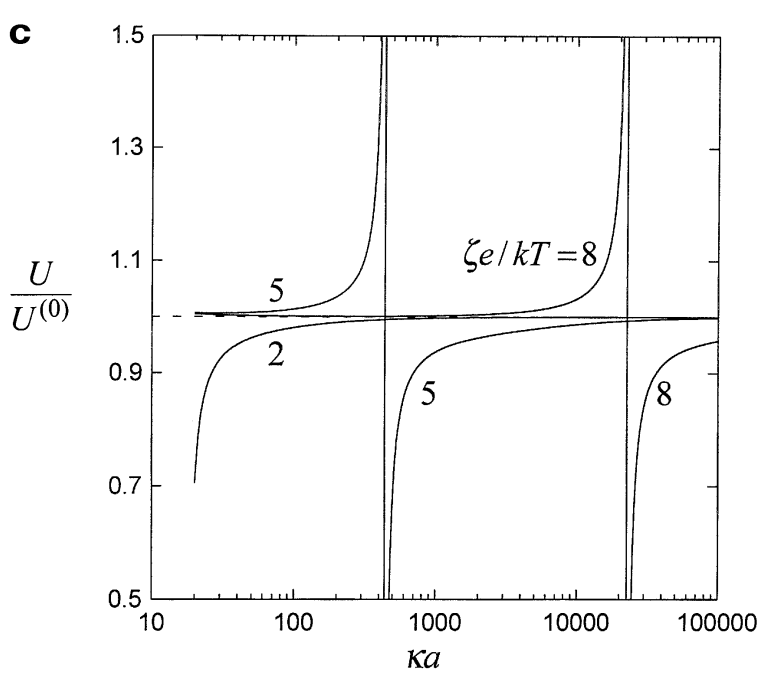

FIG . 6. Normalized translational velocities of two identical spheres undergoing diffusiophoresis perpendicular to the line through their centers versus $\kappa a$ with $\zeta e / k T$ as a parameter for the case of $f_{1}=f_{2}=0.2$ and $2 a / r_{12}=0.6$ : (a) $Z=1$; (b) $Z=2$; (c) $Z=3$. 


\section{MEAN DIFFUSIOPHORETIC VELOCITY IN BOUNDED SUSPENSIONS}

The interaction effects between pairs of arbitrarily oriented particles undergoing diffusiophoresis, obtained in Section 3, can be extended to the evaluation of the average diffusiophoretic velocity in a suspension of charged sphers. In the following, formulas for this average velocity correct to the order of first power of the volume fraction of the particles will be derived.

For a bounded suspension of particles subjected to an imposed electrolyte concentration gradient $\nabla n^{\infty}$, it is no longer possible to define the particle velocity relative to the distant fluid. Instead, the particle velocity should be calculated for a reference frame in which the net particle and fluid flux is zero and $\nabla \mu_{m}^{\infty}$ defined by Eq. [3.4] are the volume average of the electrochemical potential gradient fields over the entire suspension. Thus,

$$
\begin{gathered}
\frac{1}{V} \int_{V} \mathbf{v}(\mathbf{x}) \mathrm{d} \mathbf{x}=\mathbf{0}, \\
\frac{1}{V} \int_{V} \nabla \mu_{m}(\mathbf{x}) \mathrm{d} \mathbf{x}=\nabla \mu_{m}^{\infty},
\end{gathered}
$$

where $V$ denotes the entire volume of the suspension.

On the basis of a microscopic model of particle interactions in a dilute dispersion which involves both statistical and low Reynolds number hydrodynamic concepts $(26,27)$, the ensemble-averaged diffusiophoretic velocity of a "test" particle (denoted by the subscipt $t$ ) in a suspension of spherical particles subject to Eq. [5.1] can be expressed as

$$
\begin{aligned}
\left\langle\mathbf{U}_{\mathrm{t}}\right\rangle= & \mathbf{U}_{\mathrm{t}}^{(0)}+C\left\{\int_{V} \mathbf{v}^{*}(\mathbf{r})[g(\mathbf{r})-1] \mathrm{d} \mathbf{r}\right. \\
& +\sum_{m=1}^{2} \int_{V}\left(G_{m}\right)_{\mathrm{t}}\left[\nabla \mu_{m}^{*}(\mathbf{r})-\nabla \mu_{m}^{\infty}\right][g(\mathbf{r})-1] \mathrm{d} \mathbf{r} \\
& \left.+\int_{V} \mathbf{W}(\mathbf{r}) g(\mathbf{r}) \mathrm{d} \mathbf{r}\right\}+O\left(C^{2}\right)
\end{aligned}
$$

Here, $\mathbf{U}_{\mathrm{t}}^{(0)}=\sum_{m=1}^{2}\left(G_{m}\right)_{\mathrm{t}} \nabla \mu_{m}^{\infty}$, which is the undisturbed diffusiophoretic velocity of the test particle in a symmetric electrolyte given by Eq. [2.28], $g(\mathbf{r})$ is the radial distribution function describing the two-particle configurational probability, and $C$ is the macroscopic concentration of the neighbor particles (assumed to be identical with zeta potential $\zeta$ and radius $a) . \nabla \mu_{m}^{*}(\mathbf{r})$ and $\mathbf{v}^{*}(\mathbf{r})$ are the electrochemical potential gradient and fluid velocity fields, respectively, at position $\mathbf{r}$, when a neighboring particle at the origin $\mathbf{0}$ moves due to the prescribed concentration gradient $\nabla \mu_{m}^{\infty}$, which are expressed by Eq. [3.5] (eliminating the superscripts and subscripts) for $r \geq a$. Inside the neighboring particle $(r<a)$, both $\nabla \mu_{m}^{*}$ and $\mathbf{v}^{*}$ are constant,

$$
\nabla \mu_{m}^{*}=\nabla \mu_{m}^{\infty}+\sum_{i=1}^{2}\left(g_{m i}\right) \nabla \mu_{i}^{\infty}
$$

$$
\mathbf{v}^{*}=\sum_{m=1}^{2}\left(G_{m}\right) \nabla \mu_{m}^{\infty}
$$

Note that Eq. [5.3a] gives only the assumed quantity for $\nabla \mu_{m}^{*}$, which satisfies Laplace's equation and the continuity at the particle surface $(r=a)$ for $\mu_{m}^{*}$. W(r) is a correction function needed to account for the perturbation on $\mathbf{v}^{*}(\mathbf{r})$ owing to the presence of the test particle, and is given by

$$
\mathbf{W}(\mathbf{r})=\mathbf{U}_{\mathrm{t}}^{*}(\mathbf{r})-\mathbf{U}_{\mathrm{t}}^{(0)}-\mathbf{v}^{*}(\mathbf{r})-\sum_{m=1}^{2}\left(G_{m}\right)_{\mathrm{t}}\left[\nabla \mu_{m}^{*}(\mathbf{r})-\nabla \mu_{m}^{\infty}\right],
$$

where $\mathbf{U}_{t}^{*}(\mathbf{r})$ is the actual velocity of the test particle located at $\mathbf{r}$ with respect to the origin of a single neighboring particle at $\mathbf{0}$. $\mathbf{U}_{\mathrm{t}}^{*}(\mathbf{r})$ can be calculated from the summation of Eqs. [3.3a] and [3.8a], taking subscripts 1 and 2 to denote the test and neighboring particles, respectively. Note that the Faxen correction term involving $\nabla^{2} \mathbf{v}^{*}$, which should have appeared in Eqs. [5.2] and [5.4], equals zero, as computed from using Eq. [3.5b].

To evaluate the volume integrals in Eq. [5.2], we assume that the radial distribution function has the following equilibrium value for rigid spheres without long-range pair potential:

$$
\begin{array}{ll}
g=0 & \text { if } r<a_{\mathrm{t}}+a, \\
g=1+O(C) & \text { if } r>a_{\mathrm{t}}+a,
\end{array}
$$

where $O(C)$ is a term proportional to the concentration of neighbors. In other words, the particles must be sufficiently small so that Brownian motion dominates any multiparticle hydrodynamic interactions that might impart microscopic structure to the suspension. In general, it is necessary to obtain the pair distribution function as the solution of a conservation equation of Fokker-Planck type for a polydisperse system of spheres (28). The conditions under which the assumption of the local equilibrium is valid for a dilute dispersion consisting of different types of particles are also discussed by Reed and Anderson (27).

Given Eq. [3.5] or [5.3] for $\nabla \mu_{m}^{*}(\mathbf{r})$ and $\mathbf{v}^{*}(\mathbf{r})$, combination of Eqs. [3.3a] and [3.8a] for $\mathbf{U}_{\mathrm{t}}^{*}(\mathbf{r})$, Eq. [5.4] for $\mathbf{W}(\mathbf{r})$, and Eq. [5.5] for $g(\mathbf{r})$, the integrals in Eq. [5.2] are evaluated to obtain

$$
\left\langle\mathbf{U}_{\mathrm{t}}\right\rangle=\mathbf{U}_{\mathrm{t}}^{(0)}\left[1+\alpha_{\mathrm{t}} \varphi+O\left(\varphi^{2}\right)\right],
$$

with

$$
\begin{aligned}
\alpha_{\mathrm{t}}= & \frac{1}{A_{\mathrm{t}}}\left\{-A-2 \sum_{m=1}^{2}\left(G_{m}\right)_{\mathrm{t}}\left(b_{m}\right)+\left(\frac{a_{\mathrm{t}}}{a_{\mathrm{t}}+a}\right)^{3}\right. \\
& \times\left[-2 \sum_{m=1}^{2}\left(G_{m}\right)\left(b_{m}\right)_{\mathrm{t}}+4 \sum_{m=1}^{2} \sum_{i=1}^{2}\left(G_{m}\right)_{\mathrm{t}}\left(g_{m i}\right)\left(b_{i}\right)_{\mathrm{t}}\right. \\
& \left.\left.-12 \sum_{m=1}^{2}\left(H_{m}\right)\left(b_{m}\right)_{\mathrm{t}}-\frac{5}{2} A_{\mathrm{t}}\right]\right\}
\end{aligned}
$$


where $\varphi=4 \pi a^{3} C / 3$ is the volume fraction of the neighbor particles. The three terms in the braces of Eq. [5.7] for $\alpha_{\mathrm{t}}$ are obtained in order of the contributions from the first, second, and third integrals in Eq. [5.2]. This result is not exact, even given Eq. [5.5] holds, because $O\left(r_{12}^{-8}\right)$ terms are neglected in $\mathbf{U}_{\mathrm{t}}^{*}(\mathbf{r})$; the error will appear only in the calculation involving the correction function $\mathbf{W}(\mathbf{r})$. In the derivation of Eq. [5.7], all the neighbor particles were assumed to be identical, even though they are allowed to differ from the test particle.

For a dilute suspension of particles that have a distribution in radius and/or zeta potential, a generalization of Eqs. [5.6] and [5.7] yields

$$
\begin{aligned}
\left\langle\mathbf{U}_{k}\right\rangle= & \mathbf{U}_{k}^{(0)}\left[1+\sum_{l} \alpha_{k l} \varphi_{l}+O\left(\varphi^{2}\right)\right] \\
\alpha_{k l}= & \frac{1}{A_{k}}\left\{-A_{l}-2 \sum_{m=1}^{2}\left(G_{m}\right)_{k}\left(b_{m}\right)_{l}+\left(\frac{a_{k}}{a_{k}+a_{l}}\right)^{3}\right. \\
& \times\left[-2 \sum_{m=1}^{2}\left(G_{m}\right)_{l}\left(b_{m}\right)_{k}+4 \sum_{m=1}^{2} \sum_{i=1}^{2}\left(G_{m}\right)_{k}\left(g_{m i}\right)_{l}\left(b_{i}\right)_{k}\right. \\
& \left.\left.-12 \sum_{m=1}^{2}\left(H_{m}\right)_{l}\left(b_{m}\right)_{k}-\frac{5}{2} A_{k}\right]\right\} .
\end{aligned}
$$

Here, $\varphi=\sum_{l} \varphi_{l}$ and the subscript $k$ denotes the type of particles having radius $a_{k}$ and uniform zeta potential $\zeta_{k}$.

In a suspension of identical particles, the expression for the average diffusiophoretic velocity can be reduced from Eqs. [5.8] and [5.9] to

$$
\begin{aligned}
\langle\mathbf{U}\rangle= & \mathbf{U}^{(0)}\left[1+\alpha \varphi+O\left(\varphi^{2}\right)\right], \\
\alpha= & \frac{1}{A}\left[-A-2 \sum_{m=1}^{2} G_{m} b_{m}+\frac{1}{8}\left(-2 \sum_{m=1}^{2} G_{m} b_{m}\right.\right. \\
& \left.\left.+4 \sum_{m=1}^{2} \sum_{i=1}^{2} G_{m} g_{m i} b_{i}-12 \sum_{m=1}^{2} H_{m} b_{m}-\frac{5}{2} A\right)\right] .
\end{aligned}
$$

In the limiting situation given by Eq. [1.4] (no polarization effect in the electric double layer surrounding each particle), Eqs. [5.9] and [5.11] give $\alpha_{k l}=\alpha=-3 / 2$ and the contribution from the correction function $\mathbf{W}$ to $\alpha$ vanishes (irrespective to whether the suspension has a particle size distribution or not). The reason for a nonzero value of $\alpha$ in this limit (where there is no interaction between a pair of particles with the same zeta potential in an unbounded fluid) is that in the bounded suspension the volume-averaged flow is zero (this contributes -1 to $\alpha$ ) and the volume-averaged electrochemical potential gradients are $\nabla \mu_{m}^{\infty}$ (this contributes $-1 / 2$ to $\alpha$ ), as required by Eq. [5.1].

Results of $\alpha$ calculated from Eq. [5.11] for a suspension of identical particles at different values of $\kappa a$ and $Z$ are plotted versus $\zeta e / k T$ in Fig. 7a for a case of $D_{2}=D_{1}$. It can be seen that the mean diffusiophoretic velocity is reduced with an increase
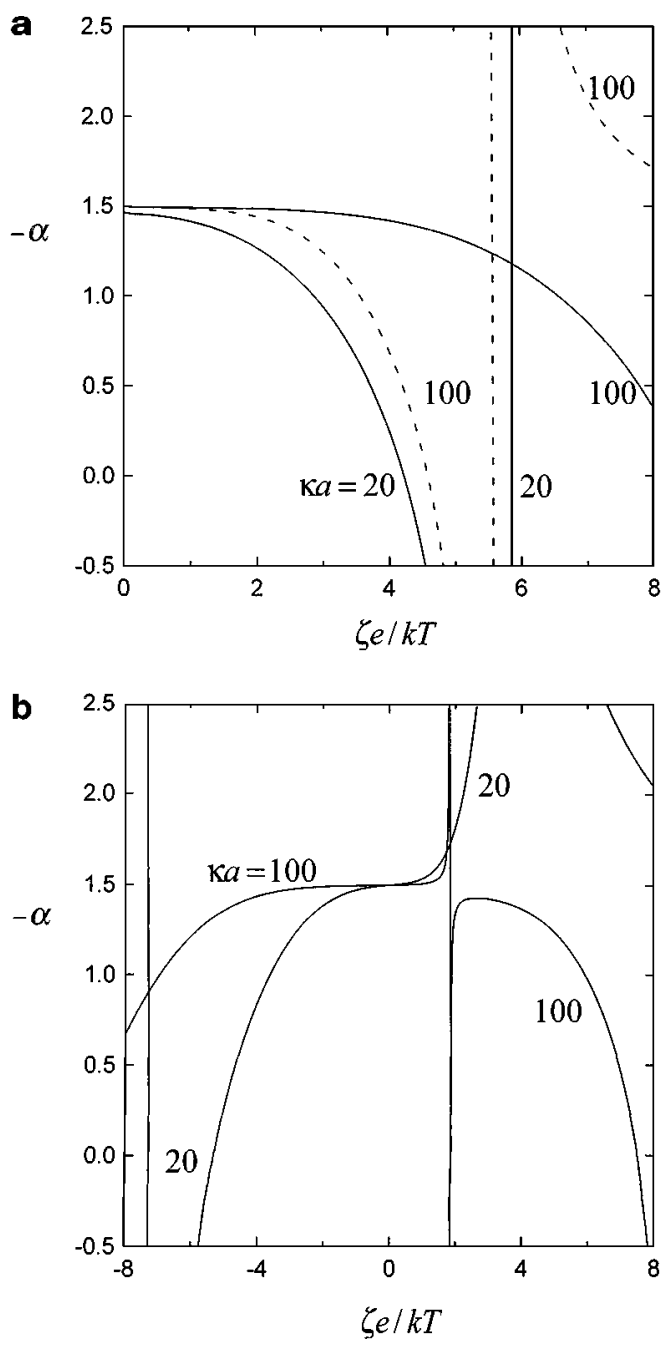

FIG. 7. Coefficient $\alpha$ evaluated from [5.11] for the diffusiophoresis of a suspension of identical spheres versus $\zeta e / k T$ : (a) $f_{1}=f_{2}=0.2$, solid curves represent the case $Z=1$ and dashed curves denote the case $Z=2$; (b) $Z=1$, $\left(D_{2}-D_{1}\right) /\left(D_{2}+D_{1}\right)=-0.2$ and $f_{1}=0.2$.

in the particle concentration (the value of $\alpha$ is negative) in the range of $\kappa a \geq 100$ and $|\zeta| e / k T \leq 8$ when $Z=1$. On the other hand, for the representative situations of $\kappa a=20$ when $Z=1$ and of $\kappa a=100$ when $Z=2$ (or 3, which is not shown in this figure), the particle velocity can be enhanced with the increase of $\varphi$ (the value of $\alpha$ can be positive) in the considered range of zeta potential and a maximum and a minimum of $\alpha$ can appear. The abrupt variation of $\alpha$ near these extremes is also due to the fact that the direction of the undisturbed velocity $\mathbf{U}^{(0)}$ reverses and its magnitude is small over there. In Fig. 7b, $\alpha$ as a function of $\zeta e / k T$ at two different values of $\kappa a$ for the case of $Z=1,\left(D_{2}-\right.$ $\left.D_{1}\right) /\left(D_{2}+D_{1}\right)=-0.2$, and $f_{1}=0.2$ is illustrated. The results for the cases $Z=2$ and 3 will give a similar dependence of $\alpha$ on $\zeta e / k T$. Again, the value of $\alpha$ can be positive or negative depending on the relevant factors.

Figure 8 shows the calculations of $\alpha$ from Eq. [5.11] for monodisperse suspensions as a function of $\kappa a$ over the range 


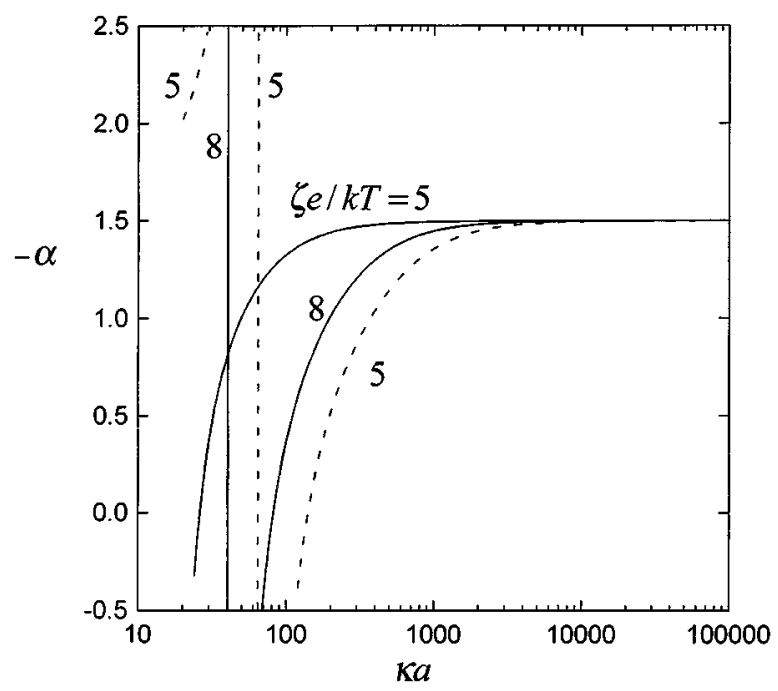

FIG. 8. Coefficient $\alpha$ evaluated from [5.11] for the diffusiophoresis of a suspension of identical spheres versus $\kappa a$ for the cases of $Z=1$ (solid curves) and $Z=2$ (dashed curves) with $f_{1}=f_{2}=0.2$.

20-10 $0^{5}$ for a case of $D_{2}=D_{1}$. As expected, $\alpha \rightarrow-3 / 2$ as $\kappa a \rightarrow \infty$ for various values of $Z$ and $\zeta e / k T$. For given values of $Z$ and $\zeta e / k T$, there can be a maximum and a minimum of $\alpha$ occurring at some $\kappa a$ and the value of $\alpha$ can be positive or negative. The plot of $\alpha$ versus $\kappa a$ for cases with $D_{2} \neq D_{1}$ will display a similar dependence as Fig. 8 .

\section{ELECTROPHORESIS IN A SYMMETRIC ELECTROLYTE}

Considered in this section is the electrophoretic motions of a single charged sphere in an arbitrary electric field, of two charged spheres in a uniform electric field, and of a dilute suspension of charged spheres. The bulk concentration $n^{\infty}$ of the symmetrically charged electrolyte beyond the electric double layers is constant. Like the analysis in the previous sections, the thickness of the double layers is assumed to be much smaller than the particles' radii and the surface-to-surface distance between the neighboring particles, but the polarization effect in the thin diffuse layers is incorporated.

First we examine the motion of a single dielectric sphere of radius $a$ with uniform zeta potential $\zeta$ in an arbitrary imposed electric field $\mathbf{E}_{A}(\mathbf{x})$. Outside the double layer, the electrochemical potentials of the ions satisfy Laplace's equation [2.1] and boundary condition [2.4] at the particle surface, but the condition [2.8] at infinity is replaced by

$$
r \rightarrow \infty: \quad \mu_{m} \rightarrow \mu_{m A}=\mu_{m}^{0}+k T \ln n^{\infty}-z_{m} e \mathbf{E}_{A} \cdot \mathbf{x}
$$

The solution for $\mu_{m}$ in this case can still be expressed as Eq. [2.19]. The governing equations, boundary conditions, and solution for the velocity field have the same forms as those given in Section 2. The final result for the electrophoretic velocity of the particle can be written as

$$
\mathbf{U}=A^{e}\left(\mathbf{E}_{A}\right)_{0},
$$

where

$$
A^{e}=\frac{\varepsilon k T}{3 \pi \eta Z e}\left[\left(2+c_{1}+c_{2}\right) \bar{\zeta}+\left(c_{1}-c_{2}\right) \ln \cosh \bar{\zeta}\right],
$$

instead of Eqs. [2.29] and [2.30] for the diffusiophoretic velocity. Analogous to the case of diffusiophoresis, this result is identical to Eq. [1.3] with $\mathbf{E}_{A}$ evaluated at the position of the particle center.

We now examine the motion of two dielectric spheres in a constant applied electric field $\mathbf{E}_{\infty}$. Similar to the case of motion of a single sphere, the formulas presented in Section 3 for the diffusiophoresis of two spheres are still valid for electrophoresis, except that condition [3.4] should be changed into

$$
\mu_{m}^{\infty}=\mu_{m}^{0}+k T \ln n^{\infty}-z_{m} e \mathbf{E}_{\infty} \cdot \mathbf{x},
$$

and the final result for the translational and angular velocities give by Eq. [3.9] is replaced by

$$
\begin{aligned}
\mathbf{U}_{1}= & A_{1}^{e} \mathbf{E}_{\infty}+\left[\frac{1}{2} A_{2}^{e}+Z e \sum_{m=1}^{2}(-1)^{m}\left(G_{m}\right)_{1}\left(c_{m}\right)_{2}\right]\left(\frac{a_{2}}{r_{12}}\right)^{3} \\
& \times(3 \mathbf{e e}-\mathbf{I}) \cdot \mathbf{E}_{\infty}+\left\{Z e \sum _ { m = 1 } ^ { 2 } \left[\frac{1}{2}(-1)^{m}\left(G_{m}\right)_{2}\left(c_{m}\right)_{1}\right.\right. \\
& \left.-\sum_{i=1}^{2}(-1)^{m}\left(G_{m}\right)_{1}\left(g_{m i}\right)_{2}\left(c_{i}\right)_{1}\right](3 \mathbf{e e}+\mathbf{I}) \\
& \left.+\left[18 Z e \sum_{m=1}^{2}(-1)^{m}\left(H_{m}\right)_{2}\left(c_{m}\right)_{1}-\frac{15}{2} A_{1}^{e}\right] \mathbf{e e}\right\} \frac{a_{1}^{3} a_{2}^{3}}{r_{12}^{6}} \cdot \mathbf{E}_{\infty} \\
& +O\left(r_{12}^{-8}\right), \\
\boldsymbol{\Omega}_{1}= & {\left[-9 Z e \sum_{m=1}^{2}(-1)^{m}\left(H_{m}\right)_{2}\left(c_{m}\right)_{1}+\frac{15}{4} A_{1}^{e}\right] \frac{a_{1}^{3} a_{2}^{3}}{r_{12}^{7}} \mathbf{e} } \\
& \times \mathbf{E}_{\infty}+O\left(r_{12}^{-9}\right) .
\end{aligned}
$$

In Eq. [6.5], the variables $\left(g_{m i}\right)_{j},\left(c_{m}\right)_{j},\left(G_{m}\right)_{j},\left(H_{m}\right)_{j}$, and $A_{j}^{e}$ denote those defined by Eqs. [2.13], [2.15], [2.26], [2.27], and [6.3], respectively, for the particle $j(j=1$ or 2).

The exact (numerical) solution for the problem of electrophoresis of two arbitrary dielectric spheres with thin but polarized double layers was obtained by using the boundary collection method $(10,17)$. Figure 9 gives a comparison of our asymptotic results of the normalized electrophoretic velocity $U_{1} / U_{1}^{(0)}$ calculated from Eqs. [6.5a] and [1.3] (illustrated by curves) for the case of two equal-sized spheres with $f_{1}=f_{2}=0.4$ and $\kappa a=100$ with the exact solution (shown by points) and indicates a good agreement between them. The errors are less than 

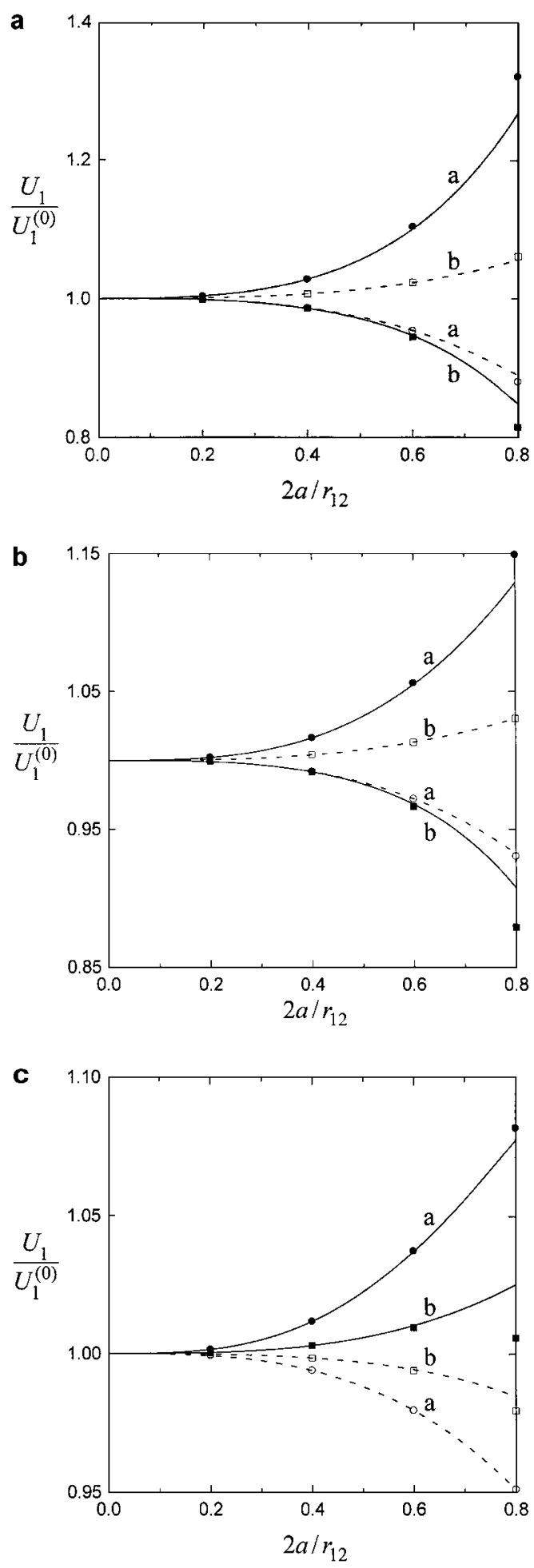

FIG. 9. Normalized electrophoretic velocity $U_{1} / U_{1}^{(0)}$ versus the separation parameter $2 a / r_{12}$ for the case of two spheres with $a_{1}=a_{2}=a, f_{1}=f_{2}=0.4$, and $\kappa a=100$ : (a) $Z=1$; (b) $Z=2$; (c) $Z=3$. The solid curves are plotted when the line through particle centers is parallel to the applied electric field, while the dashed curves are drawn when this line is perpendicular to the applied electric field. Curves "a" represent the cases with $\zeta_{1} e / k T=1$ and $\zeta_{2} e / k T=5$; curves "b" denote the cases with $\zeta_{1} e / k T=5$ and $\zeta_{2} e / k T=-5$. The points represent the exact collocation solutions.
$0.32 \%$ (or $0.07 \%$ ) for cases $2 a / r_{12} \leq 0.6$ and $4.3 \%$ (or $1.1 \%$ ) for case $2 a / r_{12} \leq 0.8$ when the two spheres are oriented parallel (or perpendicular) to the applied electric field $\mathbf{E}_{\infty}$. In the cases of two spheres having different radii, Eq. [6.5] can also be found to agree well with the exact solution. For most situations, the interaction between the two electrophoretic particles does not vary monotonically with the parameters $\zeta e / k T$ and $\kappa a$. Similar to the case of diffusiophoresis of two spheres discussed in Section 4, if the electrophoretic velocity of one sphere is enhanced (or reduced) by the other when the electric field is applied normal to the line through their centers, this velocity will be reduced (or enhanced) when the electric field is imposed parallel to the line of particle centers.

Finally, we consider the electrophoretic motion of a suspension of dielectric spheres. The analysis presented in Section 5 is also valid for this case, provided that Eq. [6.4] is used for $\mu_{m}^{\infty}$ instead of Eq. [3.4] and $\mathbf{U}^{(0)}$ represents the electrophoretic velocity given by Eq. [1.3]. With these changes, the result for $\alpha_{\mathrm{t}}$ given by Eq. [4.7] becomes

$$
\begin{aligned}
\alpha_{\mathrm{t}}= & \frac{1}{A_{\mathrm{t}}^{e}}\left\{-A^{e}+Z e \sum_{m=1}^{2}(-1)^{m}\left(G_{m}\right)_{\mathrm{t}}\left(c_{m}\right)\right. \\
& +\left(\frac{a_{\mathrm{t}}}{a_{\mathrm{t}}+a}\right)^{3}\left[Z e \sum_{m=1}^{2}(-1)^{m}\left(G_{m}\right)\left(c_{m}\right)_{\mathrm{t}}\right. \\
& -2 Z e \sum_{m=1}^{2} \sum_{i=1}^{2}(-1)^{i}\left(G_{m}\right)_{\mathrm{t}}\left(g_{m i}\right)\left(c_{i}\right)_{\mathrm{t}} \\
& \left.\left.+6 Z e \sum_{m=1}^{2}(-1)^{m}\left(H_{m}\right)\left(c_{m}\right)_{\mathrm{t}}-\frac{5}{2} A_{\mathrm{t}}^{e}\right]\right\} .
\end{aligned}
$$

The expression for $\alpha_{k l}$ and $\alpha$ in Eqs. [5.9] and [5.11] for polydisperse and monodisperse suspensions, respectively, should be altered accordingly. Analogous to the corresponding case of diffusiophoresis, Eq. [6.6] gives $\alpha_{\mathrm{t}}=\alpha_{k l}=\alpha=-3 / 2$ for a suspension of particles having the same zeta potential in the limiting situation given by Eq. [1.4]. Note that the use of the analytical formula [6.5] to evaluate $\alpha_{\mathrm{t}}$ has a great advantage over the use of the numerical solutions for the interaction between two electrophoretic spheres in that a complicated numerical integration (which usually leads to some errors, too) can be avoided in the calculation of Eq. [5.2].

Results of the coefficient $\alpha$ for the electrophoresis of a suspension of identical spheres with $D_{2}=D_{1}$ at various values of $Z, \zeta e / k T$, and $\kappa a$ calculated using Eq. [6.6] are shown in Fig. 10. Only the results at positive zeta potentials are shown since $\alpha$ is an even function of $\zeta$ here. It can be found in all cases that the average electrophoretic velocity always decreases with an increase in the particle concentration (with $-3 / 2 \leq \alpha<0$ ) and the effect of interactions between two particles (in an unbounded fluid) is to reduce the magnitude of $\alpha$. It can be seen that a maximum of $\alpha$ can exist at some $\zeta e / k T$ for a fixed value of $\kappa a$ and at some $\kappa a$ for a constant value of $\zeta e / k T$. When the value 

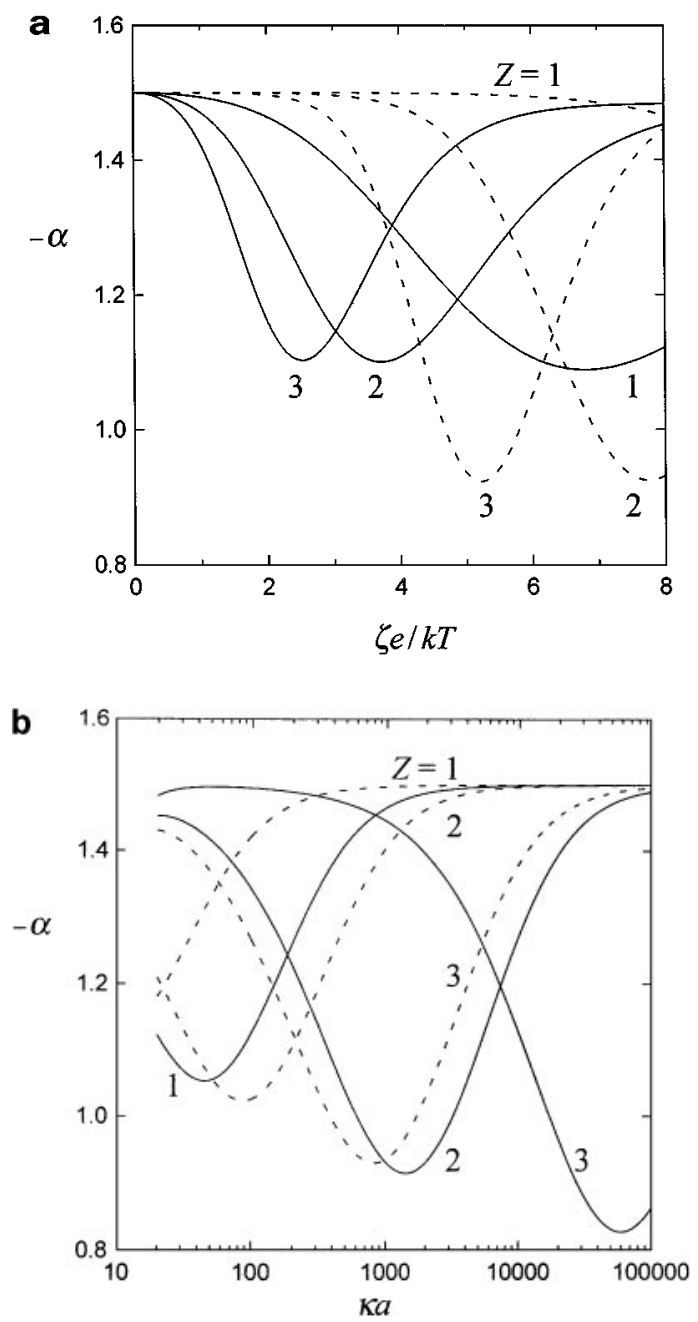

FIG. 10. Coefficient $\alpha$ evaluated from [6.6] for the electrophoresis of a suspension of identical spheres for the case of $f_{1}=f_{2}=0.2$ : (a) $\alpha$ versus $\zeta e / k T$ with $\kappa a=20$ (solid curves) and $\kappa a=1000$ (dashed curves); (b) $\alpha$ versus $\kappa a$ with $\zeta e / k T=5$ (solid curves) and $\zeta e / k T=8$ (dashed curves).

of $\kappa a$ increases, the maximum occurs at a larger zeta potential; when the value of $\zeta e / k T$ increases, the maximum occurs at a larger $\kappa a$. Also, if the counterions have a larger magnitude of valence, the locations of these maxima of $\alpha$ will shift toward smaller values of $\zeta e / k T$ or larger values of $\kappa a$.

The analytical results obtained in this section should be valid for the electrophoresis of colloidal spheres over a wide range of zeta potentials when $\kappa a>20$. For the case where the electric double layer is no longer thin with respect to the particle sizes, but the zeta potentials on the particles are low (say, Ze $\zeta / k T<$ 2 ), the electrophoretic mobilites of two interacting spheres and the average mobility of a suspension of spheres correct to first order in the volume fraction of the particles have recently been calculated numerically using the method of reflections (29) and a generalized reciprocal theorem (30). Due to the restriction of low zeta potentials (the Debye-Huckel approximation) and the neglect of the double-layer polarization effect, the normalized electrophoretic mobilities of two and a suspension of identical spheres obtained by these calculations are independent of the parameters $Z e \zeta / k T, f_{1}$, and $f_{2}$, but are functions of $\kappa a$ only.

\section{SUMMARY}

Collections of particles are often encountered in real situations of diffusiophoresis and electrophoresis. It is important to understand if the effects of particle interactions significantly affect the movement of the particles. In this work, the diffusiophoretic and electrophoretic motions of two charged spherical particles with thin but polarized electric double layers are analytically studied through the use of a method of reflections. The particles may differ in radius and/or in zeta potential at the surface, and they are oriented arbitrarily relative to the electrolyte gradient or the electric field. The results of correction to Eq. [1.5] for the diffusiophoretic velocity and to Eq. [1.3] for the electrophoretic velocity of each particle are presented in Eqs. [3.9] and [6.5], respectively, in the form of power series of $r_{12}^{-1}$ up to $O\left(r_{12}^{-7}\right)$. These reflection solutions agree well with the available exact numerical solutions obtained using the boundary collocation method. For typical cases, the errors in particle velocities are less than $0.4 \%$ for cases $2 a / r_{12} \leq 0.6$ or $4.3 \%$ for cases $2 a / r_{12} \leq 0.8$, indicating that the higher terms of $O\left(r_{12}^{-8}\right)$ are not important unless the particles get close to contact with each other. The reflection results for the interaction effects between pairs of particles are utilized to determine the mean diffusiophoretic and electrophoretic velocities in a bounded polydisperse suspension of spherical particles, based on a concept of statistical mechanics. The analytical formulas for these mean velocities, which are accurate to the first order of the volume fraction of particles of each type, are given by Eqs. [5.8], [5.9], and [6.6]. Although our analytical results are derived for the solution of a symmetric electrolyte, they can be extended to the solution containing a general electrolyte knowing its correction to Eqs. [1.3] and [1.5] for the undisturbed electrophoretic (9) and diffusiophoretic (12) velocities.

Both the analytical and the numerical solutions show that particles with the same zeta potential undergoing diffusiophoresis and electrophoresis will interact with one another, unlike the no-interaction results obtained for the limiting case assuming that the double layer surrounding each particle is infinitesimally thin. In general, the effects of particle interactions can be quite significant under appropriate conditions. For most situations, however, the particle interactions among the charged spheres are complicated functions of the properties of the particles and surrounding ions, $\zeta_{1} e / k T, \zeta_{2} e / k T, \kappa a_{1}, \kappa a_{2}, Z, f_{1}$, and $f_{2}$. Since there is no simple rule to be able to make an adequate prediction for such complicated phenomena, limited numerical solutions with interpolation and extrapolation are of little use in practical applications. In fact, these numerical solutions would often need quite a lot of computational time. Therefore, the closedform analytical results obtained here, which can be conveniently used in the calculations for various cases with $\kappa a>20$ (without the need of a computer), should be a favorable contribution to 
the evaluation and understanding of the particle interactions in diffusiophoresis and electrophoresis.

\section{ACKN OWLEDGMENT}

This research was supported by the National Science Council of the Republic of China under Grant NSC 89-2214-E002-004.

\section{REFERENCES}

1. Anderson, J. L., Annu. Rev. Fluid Mech. 21, 61 (1989).

2. Acrivos, A., Jeffrey, D. J., and Saville, D. A., J. Fluid Mech. 212, 95 (1990)

3. Keh, H. J., and Chen, S. B., Langmuir 9, 1142 (1993).

4. Henry, D. C., Proc. R. Soc. London A 133, 106 (1931).

5. Morrison, F. A., J. Colloid Interface Sci. 34, 210 (1970).

6. Dukhin, S. S., and Derjaguin, B. V., in "Surface and Colloid Science" (E. Matijevic, Ed.), Vol. 7. Wiley, New York, 1974.

7. Prieve, D. C., Anderson, J. L., Ebel, J. P., and Lowell, M. E., J. Fluid Mech. 148, 247 (1984).

8. O'Brien, R. W., and White, L. R., J. Chem. Soc. Faraday. Trans. 2 74, 1607 (1978).

9. O'Brien, R. W., J. Colloid Interface Sci. 92, 204 (1983).

10. Chen, S. B., and Keh, H. J., J. Fluid Mech. 238, 251 (1992).

11. Prieve, D. C., and Roman, R., J. Chem. Soc. Faraday. Trans. 2 83, 1287 (1987).
12. Pawar, Y., Solomentsev, Y. E., and Anderson, J. L., J. Colloid Interface Sci. 155, 488 (1993).

13. Reed, L. D., and Morrison, F. A., J. Colloid Interface Sci. 54, 117 (1976).

14. Chen, S. B., and Keh, H. J., AIChE J. 34, 1075 (1988).

15. Keh, H. J., and Yang, F. R., J. Colloid Interface Sci. 145, 362 (1991).

16. Chen, S. B., and Keh, H. J., in "Interfacial Forces and Fields" (J. Hsu, Ed.). Dekker, New York, 1999.

17. Keh, H. J., and Chen, J. B., J. Colloid Interface Sci. 158, 199 (1993).

18. Keh, H. J., and Luo, S. C., Langmuir 12, 657 (1996).

19. Levich, V. G., "Physicochemical Hydrodynamics." Prentice Hall, Englewood Cliffs, NJ, 1962.

20. Brenner, H., Chem. Eng. Sci. 19, 514 (1964).

21. Happel, J., and Brenner, H., "Low Reynolds Number Hydrodynamics." Nijhoff, The Netherlands, 1983.

22. Anderson, J. L., Int. J. Multiphase Flow 11, 813 (1985).

23. Loewenberg, M., and Davis, R. H., J. Fluid. Mech. 288, 103 (1995).

24. Velegol, D., Solomentsev, Y., Anderson, J. L., and Carnie, S. L., Colloids Surf. A 140, 59 (1998).

25. Nichols, S. C., Loewenberg, M., and Davis, R. H., J. Colloid Interface Sci. 176, 342 (1995).

26. Batchelor, G. K., J. Fluid Mech. 52, 245 (1972).

27. Reed, C. C., and Anderson, J. L., AIChE J. 26, 816 (1980).

28. Batchelor, G. K., J. Fluid Mech. 119, 379 (1982).

29. Ennis, J., and White, L. R., J. Colloid Interface Sci. 185, 157 (1997).

30. Shugai, A. A., Carnie, S. L., Chan, D. Y., and Anderson, J. L., J. Colloid Interface Sci. 191, 357 (1997). 